He...

\title{
AN ELECTRONIC DIFFERENTIAL ANALYZER
}

A. B. MACNEE

TECHNICAL REPORT NO. 90

DECEMBER 16, 1948

\section{RESEARCH LABORATORY OF ELECTRONICS}

MASSACHUSETTS INSTITUTE OF TECHNOLOGY 
The research reported in this document was made possible through support extended the Massachusetts Institute of Technology, Research Laboratory of Electronics, jointly by the Army Signal Corps, the Navy Department (Office of Naval Research) and the Air Force (Air Materiel Command), under Signal Corps Contract No. W36-039-sc-32037, Project No. 102B; Department of the Army Project No. 3-99-10-022. 


\title{
MASSACHUSETTS INSTITUTE OF TECHNOLOGY
}

Research Laboratory of Electronics

Technical Report No. 90

December 16, 1948

\section{AN ELECTRONIC DIFFERENTIAL ANALYZER}

A. B. Macnee

\begin{abstract}
An electronic differential analyzer capable of solving ordinary differential equations of orders through the sixth, both linear and nonlinear, is described. This analyzer has a high speed of operation and is extremely flexible with regard to equation parameters and initial conditions. This flexibility permits rapid investigation of wide ranges of equation solutions with regard to periodicity, instabil1ty, and discontinuities. It renders practicable the solution of endpoint boundary-value problems, that is, problems in which the final rather than inftial values are specified.

Two new computing elements, an electronic function generator and an electronic multiplier, are employed in this differential analyzer. A diode clamping circuit permits the use of a-c coupled amplifiers.

A number of representative differential equations of the linear and nonlinear types have been solved. Comparison of observed and calculated solutions reveals an accuracy of from 1 to 5 per cent, while the precision (or repeatibility) of the solutions ranges from 0.002 to 0.1 per cent. An analysis of the errors introduced into the differential-equation solutions by the frequency limitations of the computing elements, such as the integrators and adders, has been made, and the results of this analysis have been verified experimentally.
\end{abstract}




\section{AN ELECTRONIC DIFFERENTIAL ANALYZER}

\section{INTRODUCTION}

The present trend in mathematical calculators is toward large machines capable of solving more and more complicated problems with the greatest accuracies (1)(2)(3). These large machines are extremely expensive to build and operate; the use of the M.I.T. Differential Analyzer for an elght-hour day, for example, costs about $\$ 400$. This high cost, together with the limited number available, has prevented many investigators from realizing the advantages of these machines.

Early in the Fall of 1945 it was felt that there was considerable need for a differential analyzer of somewhat different characteristics from any then in existence or under development*. There appeared to be the need for a machine having the following characteristics: (a) moderate accuracy, of perhaps 1 to 10 per cent, (b) much lower cost than existing differential analyzers, (c) the ability to handle every type of ordinary differential equation, (d) high speed of operation, (e) above all, extreme flexibility in order to permit the rapid investigation of wide ranges of equation parameters and initial conditions.

A differential analyzer of this type bears the same relation to the larger differential analyzers which a slide rule bears to a desk calculating machine. Its uses are numerous. (a) It can be used, as a slide rule is used, to give rapld solutions of moderate accuracy to the differential equations encountered by the engineer, physicist, and mathematician. In this role it is valuable both in solving higher-order ordinary differential equations with constant coefficlents, which are very tedious to handle analytically, and also nonlinear equations and equations with variable coefficients which often can not be solved at all analytically. (b) Such a differential analyzer can also be used as an adjunct to one of the larger differential analyzers. It is used then to carry out the time-consuming exploratory solutions necessary to determine the ranges of equation parameters and initial conditions of interest. This preliminary work can be done at a great saving in time and money, and then, if warranted, the larger and more accurate machine can be used to obtain the final desired solution. (c) Such a differential analyzer, by virtue of its moderate cost and great flexibility, is very useful as a teaching tool in the fields of mathematics, engineering, and physics.

In the author's opinion, the objectives of flexibility, moderate cost and high-speed operation are most easily achieved with an all-electronic machine.

*This need was first polnted out to the author by Prof. Henry Waliman, Department of Mathematics, Massachusetts Institute of Technology, who'suggested development of such a machine. The author wishes to express to prof. Wallman his deepest appreclation for his guidance and encouragement of this work. 
It is important to realize that the main advantage of the high speed of this analyzer is that it permits rapid exploration of a wide range of solutions. In a fundamental way this is perhaps the most significant advantage of the electronic analyzer over other differential analyzers.

There are many important problems involving the solution of a differential equation in which the crux of the matter is to find the initial conditions for which the solutions (a) are stable, or periodic, or continuous, or (b) satisfy certain specified final conditions. Such problems frequently require exploration of 1000 different solutions (e.g., 10 values for each of three parameters) and might well represent a prohibitive investment of time on a slow differential analyzer which requires several minutes for each solution; on the present electronic analyzer such an exploration can be carried out in an hour or two. An example of this sort (the solution of the equation $d^{3} y / d t^{3}-y\left(t-t_{0}\right) / 4=0$ over the range $0 ₹ t ₹ t_{0}$ for specified final conditions) is given in Section $V$, page 36.

For problems of this type, the usefulness of the electronic analyzer actually surpasses that of the more elaborate and accurate, but slower, mechanical differential analyzers.

\section{THE ELECTRONIC DIFFERENTIAL ANALYZER SYSTEM}

\section{A. Necessary Bas1c Computing Elements}

The electrohic differential analyzer described here* is of the continuous-variable type, utilizing the basic feedback principle which was first applied to differential analyzers by Vannevar Bush (4). It employs voltages as the dependent variables and tine as the independent varlable; the basic functional operation of this analyzer is time integration.**

The computing elements required by the electronic differential analyzer can be determined by consldering a typical differential equation. An ordinary differential equation with constant coefficients can be written in the form

$$
\sum_{n=0}^{m} A_{n} \frac{d^{n} y}{d t^{n}}=F(t), \quad(n=1,2, \ldots, m),
$$

\footnotetext{
"ThIs work $1 \mathrm{~s}$ described In greater getail In an M.I.T. doctorate thesis "An Flectronic Differential Analyzer" by A.B.Macne, submitted to the Electrical Engineering Department, September, 1948.

* Iimitations are imposed on time differentiators by amplifier bandwiaths; therefore a general electronic differential analyzer cannot be designed using differentiators as basic unitsi in particular, differential equations whose characteristic roots have positive real parts cannot be solved with practical differentiators.
} 
where

$F(t)$ is an arbitrary driving function of time,

the coefficients $A_{n}$ are constants, and

$m$ is the order of the differential equation.

For the purpose of solution on the differential analyzer the equation is rewritten by 1solating the highest derivative:

$$
\frac{d^{m} y}{d t^{m}}=-\frac{1}{A_{m}} \sum_{n=0}^{m-1} A_{n} \frac{d^{n} y}{d t^{n}}+\frac{F(t)}{A_{m}} \text {. }
$$

A block diagram of the general setup to solve this equation with the electronic differential analyzer is given in Figure 1.

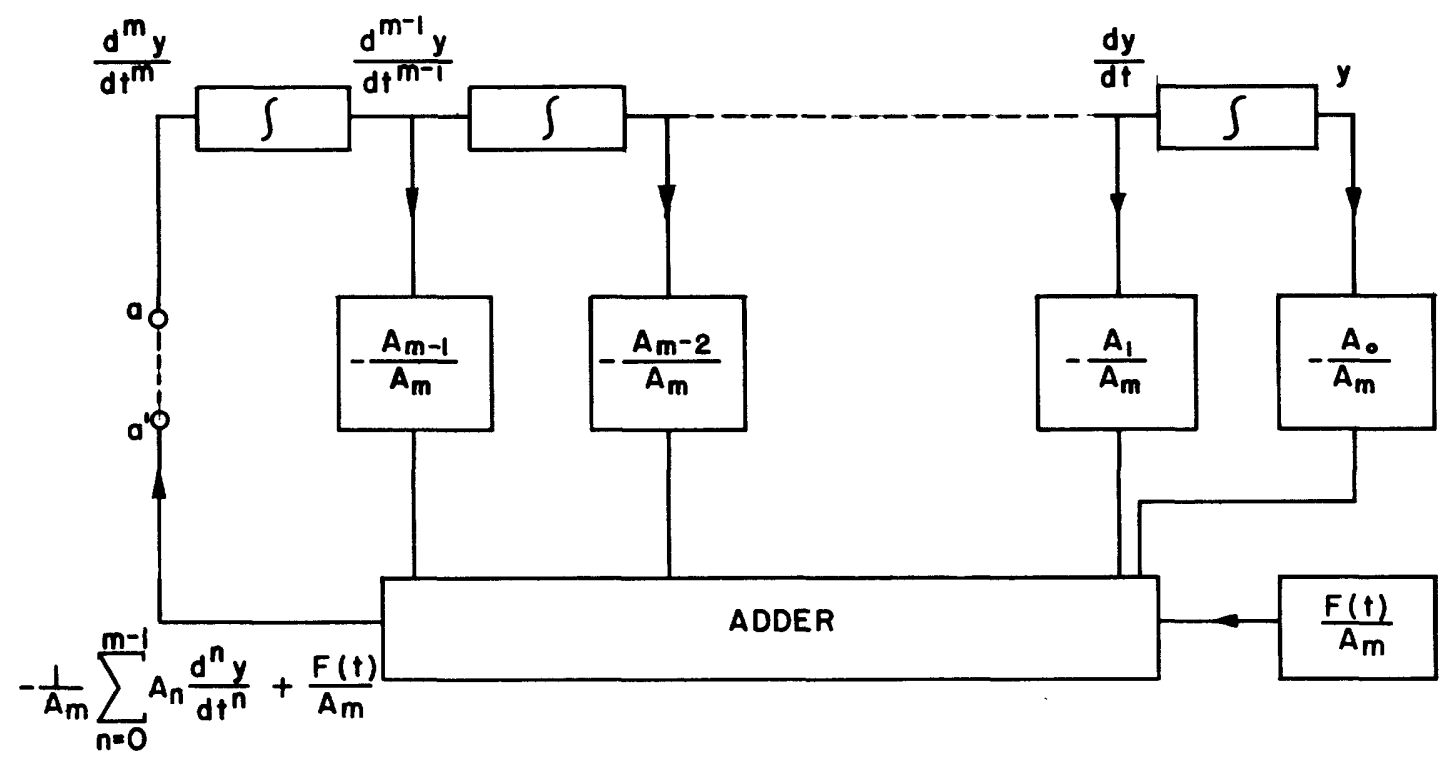

Fig. I Block diagram of setup for the solution of an ordinary linear differential equation of order m with constant coefficients.

A voltage proportional to $\mathrm{d}^{\mathrm{m}} \mathrm{y} / \mathrm{dt} \mathrm{t}^{\mathrm{m}}$ is assumed to exist at the terminal a in Figure 1. The differential analyzer units are then interconnected to generate from $\mathrm{d}^{\mathrm{m}} \mathrm{J} / \mathrm{dt}^{\mathrm{m}}$ all the terms on the right-hand side of Eq.(2); these terms appear at the terminal $a^{\prime}$ in Figure 1. Connection of the terminals $a-a^{\prime}$ then satisfies the differential equation (2), and the solution is obtained by observing the voltage proportional to $\mathrm{y}$ on a cathode-ray oscilloscope.

The electronic computing elements used to solve Eq.(2) are, as indicated in Figure 1.

1. time-integrators, 1.e., units to integrate a voltage with respect to time, 
2. amplifiers for the constant coefficients,

3. an adder, and

4. a function generator to generate the desired driving function $F(t) / A_{m}$.

If the electronic differential analyzer is to handle any ordinary differential equation including those of the variable-coefficlent and nonlinear types, two additional units are required: these are

5. multipliers, and

6. arbitrary function generators, units to generate an arbitrary function of the dependent, as well as the independent, variable.

\section{B. Solution Display}

The electronic differential analjzer presents 1 ts solutions on a cathode-ray osc1lloscope. Usually the solution is plotted against time, in which case a perlodic voltage varying linearly with time (a saw-tooth wave) is applied to the horizontal deflecting system, and the voltage corresponding to the desired solution in the differential analyzer setup is applied to the vertical deflecting system. Sometimes the solution is plotted against a time-dependent variable; in this case the suitable time-dependent voltage is applied to the horizontal plates.

\section{Repetition Rate}

In order that the solution displayed on the cathode-ray-tube face appear as a stationary curve to the observer's eye, it is necessary that the solutions traced on the screen be repeated at a reasonably high rate. If the cathode-ray tube employs the common $\mathrm{P}-1$ phosphor, this repetition rate should be at least 30 per second. If one of the more persistent screens, such as the P-7 phosphor, were used, a repetition rate as low as $1 \mathrm{cps}$ might be employed*. In the differential analyzer described here the repetition rate is $60 \mathrm{cps}$, permitting use of the a-c power mains as a standard source of synchronizing signals.

\section{Sequence of Operation}

For the repetition rate employed, sixty new solutions are thus presented every second, superimposed one upon the other on the face of the output cathode-ray tube. In order that this display appear as a single line on the

* Component IImitations at high and low frequencies introduce errors into solutions obtained on an electronic differential analyzer. The cholce of repetition rate is a compromise; a high repetition rate requires excellent high-frequency response for ali analyzer components while a low repetition rate imposes stringent low-frequency response requirements. 
output screen, it is necessary that each solution be identical with its predecessor. Th1s imposes the requirement that the infital conditions of each solution be identical with those of the preceding solution. In general, the various component voltages in the differential analyzer at the end of a solution will not be the same as those required by the inftial conditions; It is therefore necessary to allow some time between the end of each solution and the beginning of the next solution to permit the reestablishment of the proper initial conditions. In the electronic differential analyzer this is accomplished by allotting approximately $1 / 120$ of a second for the solution of the differential equation and $1 / 120$ of a second for the reestablishing of the initial values prior to the next solution. Figure 2 is a sketch of the

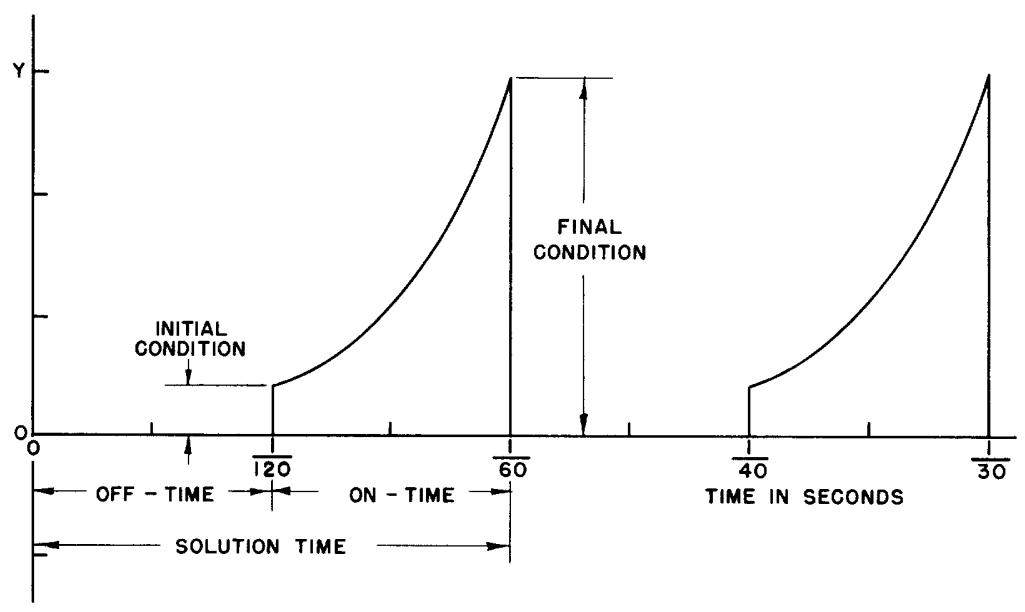

Fig.2 Appearance of a typical differential-analyzer solution as a function of time.

solution of a typical equation as it appears in this analyzer if two complete solution periods are displayed. Figures $3(a)$ and $3(b)$ are photographs of observed solutions of the differential equation

$$
\frac{d y}{d t}=k y \text {. }
$$

Figure 3(a) displays two complete solution periods, showing both the onand off-times. Figure $3(b)$ gives the solution as normally displayed in the differential analyzer; here a blanking voltage has been applied to the intensity grid of the cathode-ray tube during the off-time of the solution period so that only the solution is displayed.

\section{E. Initial Conditions}

To satisfy the inftial conditions of the difierential equation requires establishment of specified initial values of all voltages throughout the differential analyzer at the beginning of each solution period. This is accomplished by forcing all voltages in the analyzer to a constant reference 


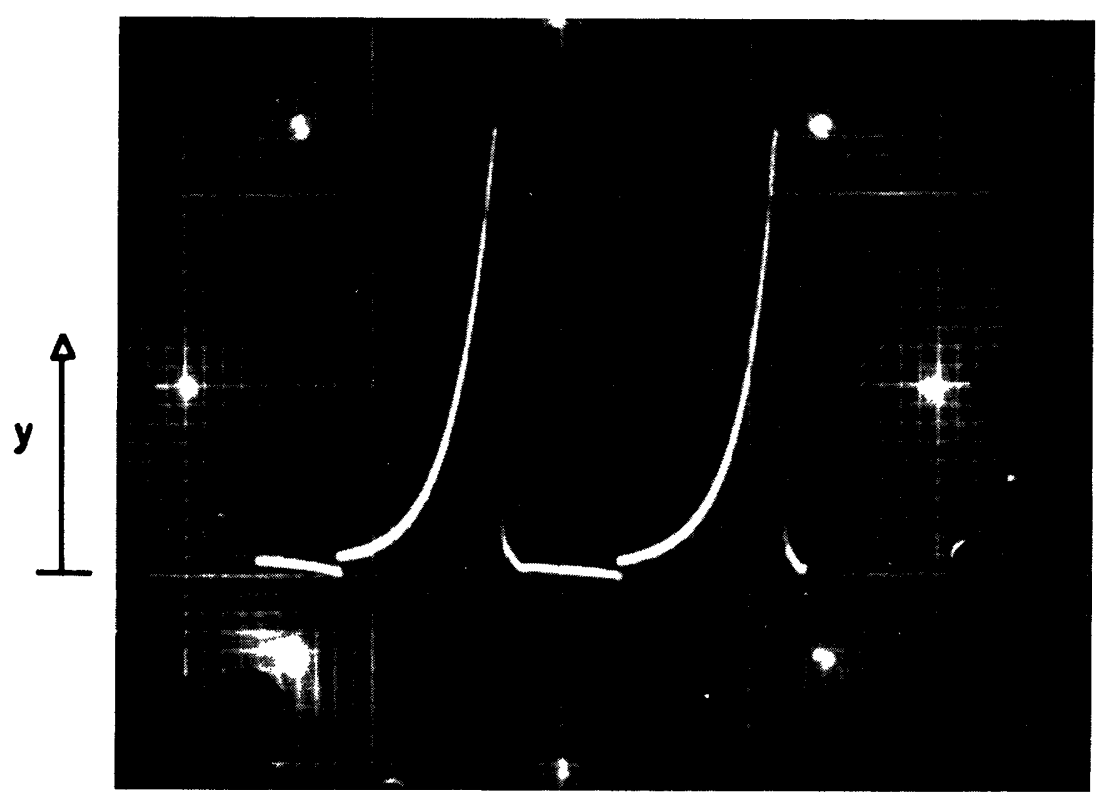

(a)

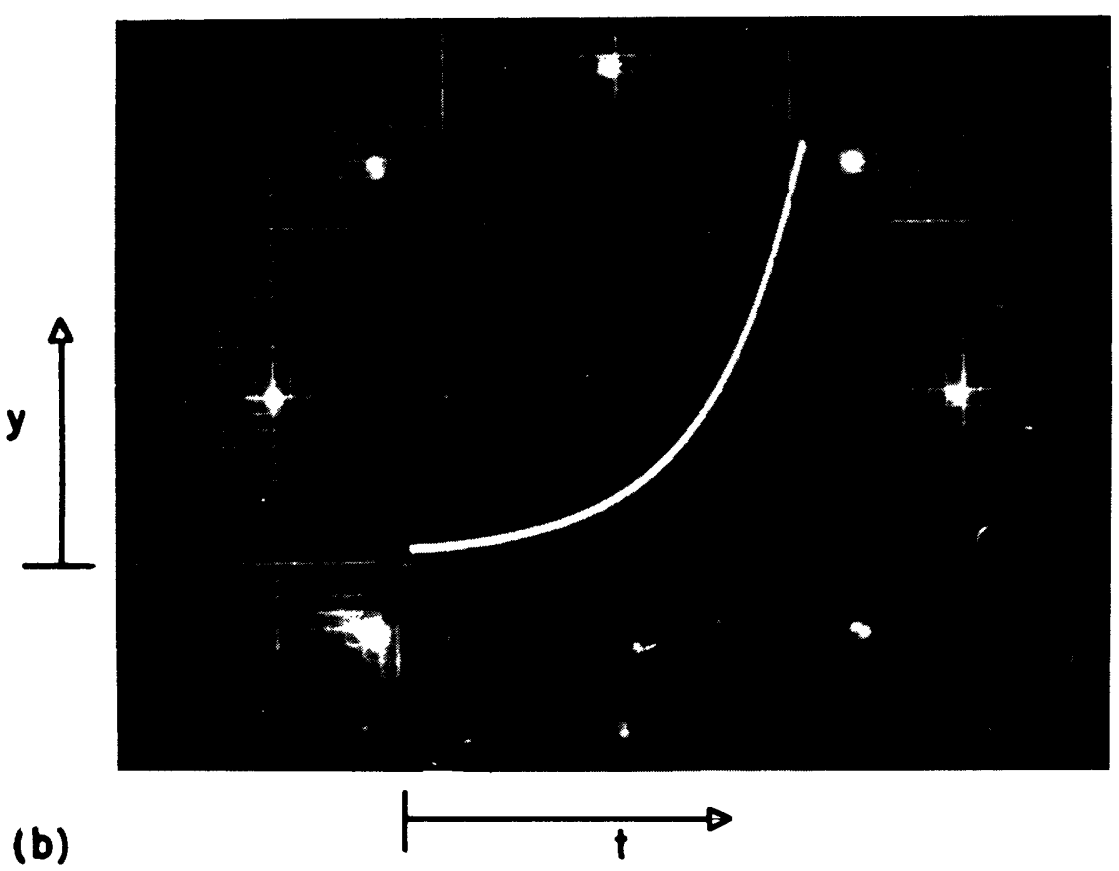

Fig.3(a) Two complete periods of the solution of the equation $\mathrm{dy} / \mathrm{dt}=\mathrm{ky}$.
Fig.3(b) Solution of $\mathrm{dy} / \mathrm{dt}=\mathrm{ky}$ as normally displayed with the off period blanked out by an intensity gate.

level, usualig zero volts, during the off-time. At the beginning of each on-time for the differential analyzer, voltage steps of proper magnitude are added to the output of each integrating unit to set the desired initial values. These "Initial value" voltage steps remain constant throughout the solution time; they are adjustable by the operator by means of potentiometers. 
As the operator varies the initial conditions, he sees instantaneously the effect of his adjustment because $a$ new solution is traced every $1 / 60$ of a second. Figure 4 is a triple-exposure photograph of the solution of Eq. (3) for three different initial values.

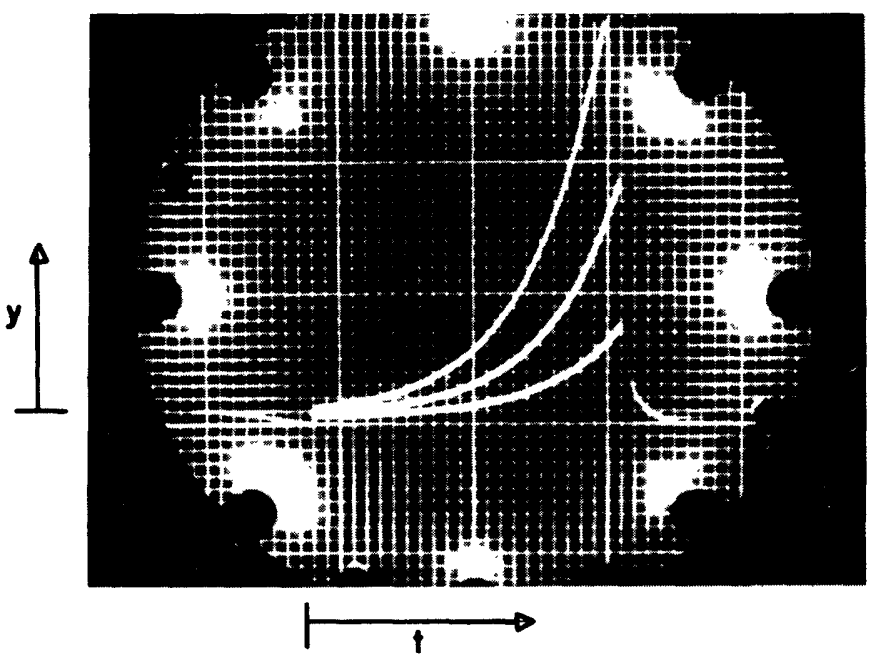

Fig.4 Triple-exposure photograph of the solutions of $\mathrm{dy} / \mathrm{at}=\mathrm{ky}$ for three values of $\mathrm{y}_{0}$.

III. COMPUTING ELEMENTS

The differential analyzer system outlined in Section II requires two new elements, an electronic multiplier and an electronic function generator; it also requires a switching or gating scheme for the time-integrating and adding clrcults. The detalls of some of the computing elements are discussed in this section. Section IV considers the principal sources of error encountered in this electronic differential analyzer. Some of the results achieved with the complete differential analyzer are described in Section $V$. The reader who is not interested in the constructional detalls of the electronic differential analyzer can omit Sections III and IV without loss of continuity.

A. Mult1plier

It was necessary to develop a new multiplying circuit for the electronic differential analyzer because, although considerable work had been done on electronic multiplication, none of the existing multiplying circuits appeared to satisfy the requirements of four-quadrant operation together with the necessary high speed of response $(5)(6)(7)(8)$. Four-quadrant operation permits handling of inputs of both algebraic signs. For the present electronlc differential analyzer, the on-time is 8,300 microseconds, as indicated in Figure 2. Lack of speed in that channel of the multiplier which is connected Into the differential-analyzer feedback loop can lead to serious errors, as discussed in Section IV-D. A rise time of about 5 microseconds 
has been found adequate for most problems considered. For many problems, extreme speed is required in only a single channel of the multiplier. Such a situation arises, for example, in equations with variable coefficients if the rate of change of the coefficlent is much less than the rate of change of the solution.

B. The Crossed-Fields Electron-Beam Multiplier

Because of the high-speed operation of the electronic differential analyzer, it was necessary to develop a new electronic multiplier. This multiplier, which employs an electrostatic-deflection cathode-ray tube, will be called the crossed-fields electron-beam multiplier. Figure 5 is a sketch of the geometry of a typical electrostatic-deflection cathode-ray tube. The electron beam leaves the electron gun with the velocity $\bar{k} v_{z} \cdot(\overline{1}, \bar{j}, \bar{k}$, are unit vectors in the $x, y, z$ directions, respectively.) If there is a potential, $E_{1}$, between the first pair of deflecting plates (the $y$-plates), the electrons passing between these plates are given a component of velocity, $\bar{j} v_{y}$, in the $y$-direction, according to the relation

$$
\bar{j} v_{y} \propto \bar{j} \frac{E_{I}}{v_{z}} .
$$

The electron beam is then moving in the region a-a'. With the velocity

$$
\bar{v}=\vec{k} v_{z}+\bar{j}_{y} .
$$

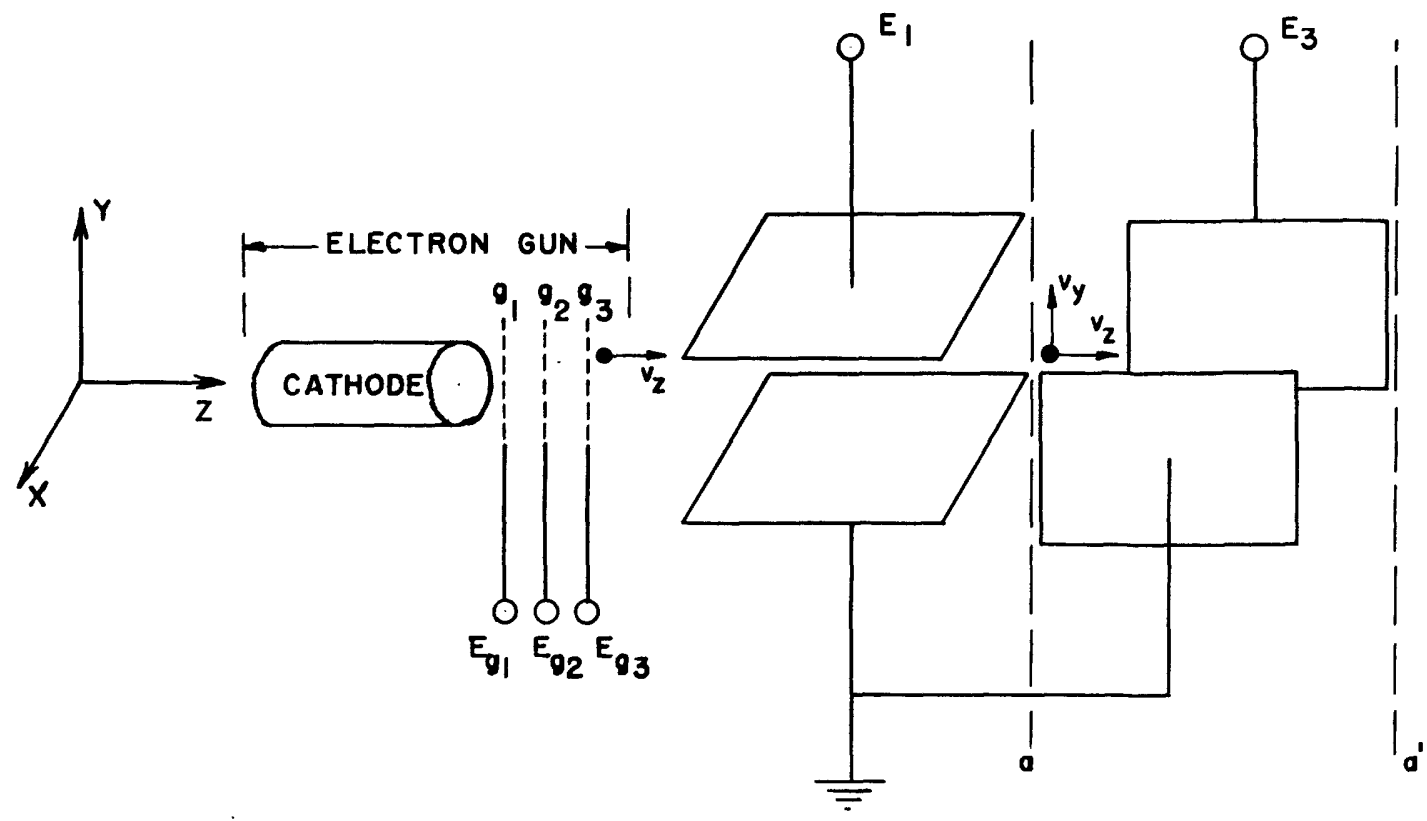

Fig.5 Geometry of a typical electrostatic-deflection cathode-ray tube. 
Now suppose there is a potential, $\mathrm{E}_{3}$, between the $x$-plates. In the region $a-a^{\prime}$ there is a force, $\bar{F}_{x e}$, acting ${ }^{3}$ on the electrons in the $x$-direction, due to the electrostatic field between the $x$-deflecting plates,

$$
\overrightarrow{\mathrm{F}}_{\mathrm{xe}}=\mathrm{e} \bar{\varepsilon}_{\mathrm{x}} \text {, }
$$

where $e=$ the electron charge, $1.60 \times 10^{-19}$ coulombs, and

$\bar{\varepsilon}_{\mathrm{x}}=$ the electrostatic field between the $x$-deflecting plates due to the impressed voltage $\mathrm{E}_{3}$.

$$
\left|\bar{\varepsilon}_{\mathrm{x}}\right| \propto_{3} \mathrm{E}_{3}
$$

If an axial magnetic field, $\overline{\mathrm{K}}_{z}$, is also present in the region $a-a^{\prime}$ (generated, for example, by a coll so wound as to have its axis of symetry ccincident with the z-axis of the cathode-ray tube), there is an additional force, $\overline{\mathrm{F}}_{\mathrm{xm}}$, exerted on the electron beam,

$$
\bar{F}_{x m}=e\left(\overline{j v}_{y} \times \bar{k}_{z}\right) \text {, }
$$

where the $x$ denotes vector cross product. This axial magnetic field is conveniently generated by a coll wound with its axis of symmetry colncldent with the $x$-axis of the cathode-ray tube; then for a coll current $I_{2}$

$$
\mathrm{B}_{\mathrm{z}} \propto \mathrm{I}_{2}
$$

An important point is that the vector $\overrightarrow{\mathrm{F}}_{\mathrm{Xm}}$ points in the $\mathrm{x}$-direction.

The speed $v_{y}$ is proportional to $E_{l}$, and the coll current $I_{2}$ can be made proportional to some other external voltage $\mathrm{E}_{2}$; then one finds from Eqs. (4) (8) and (9) that

$$
\overline{\mathrm{F}}_{\mathrm{xm}} \propto \mathrm{E}_{1} \cdot \mathrm{E}_{2}
$$

Equations (6) and (8) show that in the region $a-a^{\prime}$ of Figure 5 there are two forces $\vec{F}_{x e}$ and $\vec{F}_{x m}$ acting on the electron beam in the $x$-direction because of the crossed electric and magnetic fields.

If some means is available to make these two forces equal and opposite, then

$$
\bar{\varepsilon}_{x}=-\left(\overline{j v}_{y} \times \overline{k B}_{z}\right)
$$

and from Eqs. (7) and (10) one finds that

$$
E_{3}=k\left(E_{1} \cdot E_{2}\right) \text {; }
$$

the voltage $\mathrm{E}_{3}$ is thus proportional to the product of the two input voltages $\mathrm{E}_{1}$ and $\mathrm{E}_{2}$.

In the arrangement described above, the only forces acting to deflect the electron beam in the $x$-direction are those in the region $a-a '$. If these forces add up to zero, there is no $x$-deflection of the electron beam. 
This fact can be used to bring about the desired equality of the forces $\overrightarrow{\mathrm{F}}_{\mathrm{xe}}$ and $-\overline{\mathrm{F}}_{\mathrm{xm}}$, as indicated in Figure 6 .

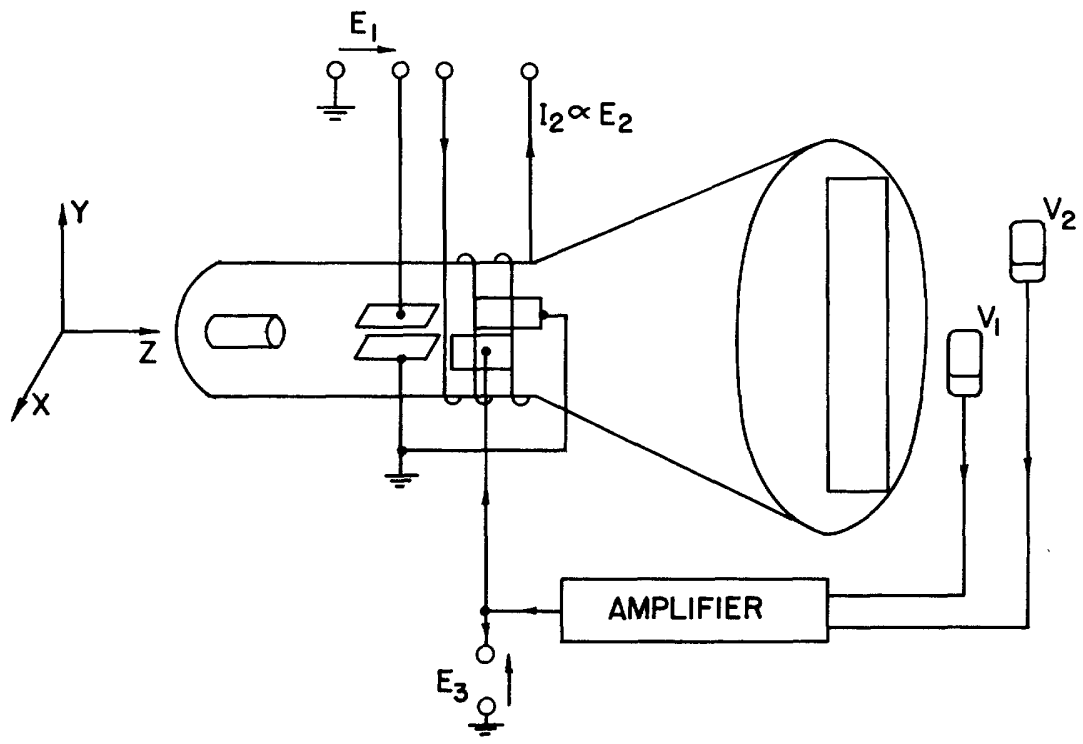

F1g.6 Crossed-fields electron-beam multiplier.

At the face of the cathode-ray-tube screen, two photocells, $\mathrm{v}_{1}$ and $\mathrm{v}_{2}$, are located. Between these photocells a vertical partition is placed colncldent with the $y$-axis of the cathode-ray tube. If no $x$-forces act on the electron beam, it will strike somewhere along the edge of this partition. The outputs of the two photocells are subtracted and fed to an amplifier which is connected to the electrostatic $x$-deflecting plates. For no $x$-deflection, the outputs of the two photocells are equal and opposite, so that there is no voltage $\mathrm{E}_{3}$. Th1s is the case when the magnetic force $F_{x m}$ is zero, that is, when either $B_{z}$ or $v_{y}$ is zero. If both $B_{z}$ and $v_{Y}$ are non-zero, there is a magnetic force; and the electron beam is deflected to the right or left of the partition on the output screen, depending upon the relative signs of the two inputs $E_{1}$ and $E_{2}$. Such a motion of the electron beam results in increased output for one photocell and decreased output from the other. The difference of these outputs is fed back to the electrostatic plates as $\mathrm{E}_{3}$ with the proper phase to oppose the magnetic force. If the gain around the feedback loop is made sufficlently large, the feedback voltage is proportional to the magnetic force and thus to the product $E_{1} \cdot E_{2} \cdot$ The magnitude of the magnetic force $\bar{F}_{x m}$ is proportional to the product of $E_{1}$ and $E_{2}$, and the force changes direction when either $E_{1}$ or $E_{2}$ change sign; therefore, four-quadrant multiplication is obtained. 
C. Measured Characteristics of the Crossed-Fields Multiplier

The speed of response of this multiplier is determined by (a) the speed with which the voltage $\mathrm{E}_{1}$ across the $\mathrm{y}$-deflecting plates can be varled, (b) the speed with which the magnetic field $\mathrm{B}_{z}$ can be changed, and (c) the speed with which the feedback loop responds. For the multiplier of this type used in the present electronic differential analyzer, the rise time of the output voltage $\mathrm{E}_{3}$ when a step voltage 1s applied at $\mathrm{E}_{1}$ (See Fig. 6) is from 1 to 6 microseconds, according to the amplitude of the input. The rise time changes with amplitude because the photocell error-sensing system is nonlinear for large error signals. * The rise time for inputs applied at $\mathrm{E}_{2}$ (F18. 6) is considerably slower, being about 80 microseconds. This could be improved, if required, by redesigning the amplifier supplying current to the magnetic field coll.

An important feature of a multiplier is its usable dynamic range. This is controlled in a four-quadrant multiplier by the zero balances. These zero balances limit the degree to which the multiplier output is zero when one input is zero and the other maximum. The experimentally observed balance characteristic of the crossed-fields multiplier is

$$
E_{3}=k\left(E_{1} \cdot E_{2}+.009 E_{1}+.015 E_{2}+.0005\right) \text {, }
$$

where

$E_{1}$ is the input controlling the $y$-deflection, normalized to a maximum value of unity, and

$\mathrm{E}_{2}$ is the voltage controlling the coil current, normalized to a maximum value of unity.

The residual output observed when $\mathrm{E}_{2}=0$ is caused by (a) stray magnetic fields and (b) lack of exact coincidence between the partition in Figure 6 and the zero $x$-deflection line on the cathode-ray-tube face. Better magnetic shielding, together with more accurate positioning of the photocell pickup unit, would probably reduce this output by a factor of ten.

The residual output observed for $E_{1}=0$ appears to be caused primarily by defocusing effects when the magnetic field $B_{z}$ is large. The limitation on the magnitude of the output voltage is the point at which the electron beam strikes the $x$-deflecting plates. The nonlinear distortion of this multiplier with a steady sinusoidal signal on the input is less than 2 per cent at the maximum output.

Figure 7 is an oscilloscope photograph of the multiplier output when

\footnotetext{
*This nonlinearlty could be removed by replacing the partition shown in Figure 6 with a strip of photographic film having a density varying from compiete opacity along the center line to transparency along the eages of the cathode-ray-tube screen.
} 
the inputs are two sinusoidal signals having frequencies of 60 and 480 cps respectively.

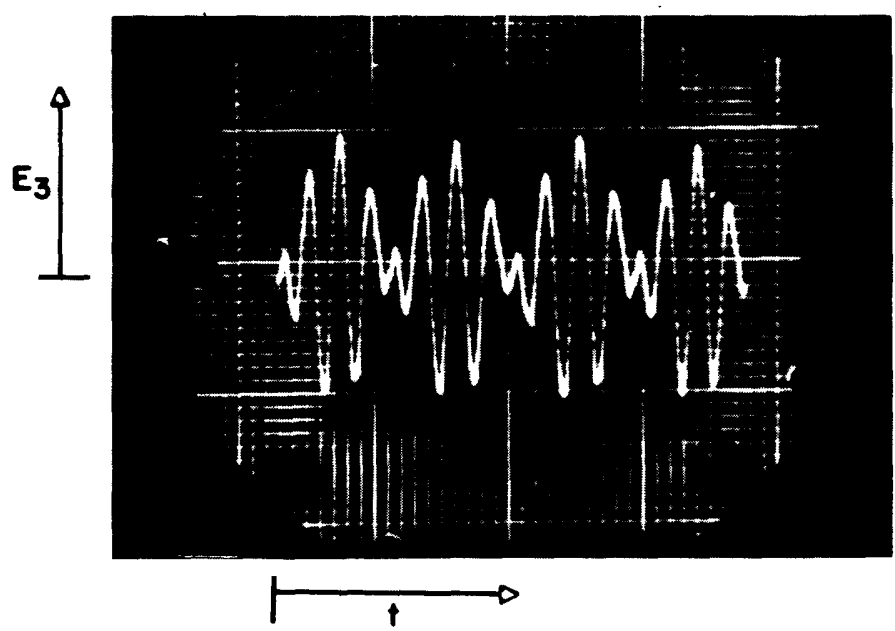

Fig. 7 Output of the crossedflelds electron-beam multiplier versus time; $E_{1}$ is a 480 -cps sinusoidal voltage and $E_{2}$ a 60 -cps sinusoidal voltage.

\section{Modification of the Crossed-Fields Multiplier for Division}

The crossed-fields multiplier can be modified to perform the operation of division, without the use of any additional computing components. If the amplified output from the pickup photocells is applied to the vertical rather than to the horizontal plates of the cathode-ray tube (Figure 6), then the feedback voltage is $E_{1}$, and $E_{3}$ becomes an input voltage. By controlling $v_{y}$, the feedback loop can st1ll keep the two forces $\bar{F}_{x e}$ and $\bar{F}_{x m}$ equal in magnitude and opposite in s1gn, so Eq. (12) is still valid. Since the output voltage is now $E_{1}$, this equation is rewritten

$$
E_{1}=\frac{E_{3}}{\mathrm{KE}_{2}}
$$

the voltage $E_{1}$ is thus proportional to the quotient of the two input voltages $E_{2}$ and $E_{3}$.

\section{E. Arbitrary Function Generator}

To generate an arbitrary function, the mechanical differential analyzers utilize an input table. The desired function is drawn on a table and an operator manually tracks this curve with a cross-hair as the machine moves. For the electronic differential analyzer described here, this tracking procedure must be performed automatically and at very high speeds.

The function generator used in this electronic differential analyzer is shown in Figure 8. This circuit was developed by the author in the summer of 1947. It has been described independently by other investigators in this country and England (9)(10)(11).

As Figure 8 shows, the arbitrary function is cut out of some opaque material in the form of a mask. This mask is placed across the face of the 


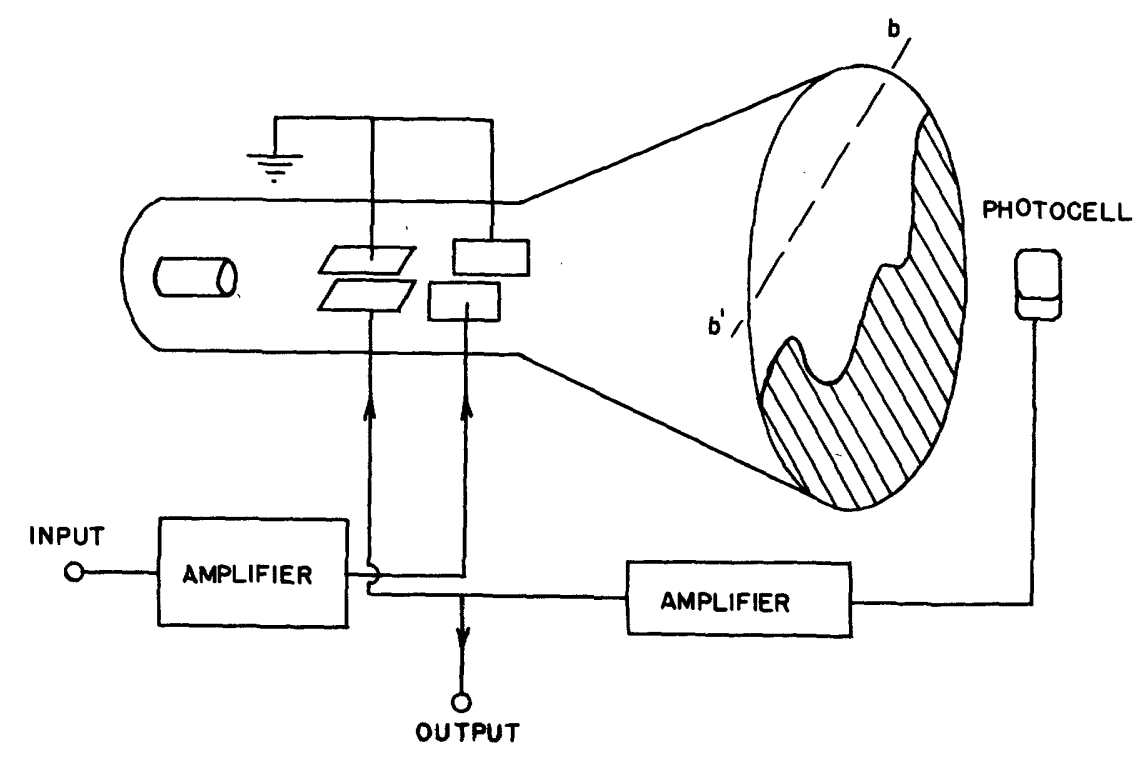

Fig. 8 Feedback arbitraryfunction generator.

cathode-ray tube. The output of a photocell located in front of the masked cathode-ray.tube screen is fed through an amplifier to the vertical deflecting system of the tube. The phase of this amplifier is chosen to give a downward deflection of the cathode-ray-tube beam when the amount of light striking the photocell is increasing. A biasing voltage at the output of this amplifier is so chosen that if no light enters the photocell, the electron beam strikes the screen along the line $b-b^{\prime}$. The line $b-b^{\prime}$ is everywhere above the function mask. If the feedback loop is now closed, then, so long as the electron beam strikes the screen above the function mask, the photocell develops an output forcing the electron beam down toward the mask. As the electron beam reaches the mask, the light spot on the screen begins to be obscured by the edge of the mask; as a result, the light striking the photocell, and correspondingly the voltage forcing the electron beam downward, is reduced. The beam takes up an equilibrium position such that the light striking the photocell generates just enough voltage at the amplifier output to hold the beam stationary.

If the electron beam is now moved in a horizontal direction by a voltage applied to the horizontal deflecting system, the beam is constrained to follow the silhouetted function mask at every point. Because the horizontal position of the electron beam and the input to the horizontal deflecting amplifier are proportional, the output of the feedback loop is the plotted function of the input voltage.

F. Measured Characteristics of Arbitrary Function Generator

The measured characteristics of the arbitrary function generator used in this electronic differential analyzer are tabulated below: 
1. maximum output rate of change $=0.073$ to 0.11 volts $/ \mathrm{mlcrosecond}$,

2. maximum output voltage $=2$ volts, and

3. maximum nonlinear distortion $=2.5 \%$.

Figure 9 (a) shows a staircase test pattern used in testing the speed of the arbitrary function generator, and Figure $9(b)$ shows a photograph of the output of the function generator when generating the test function*.

(a)
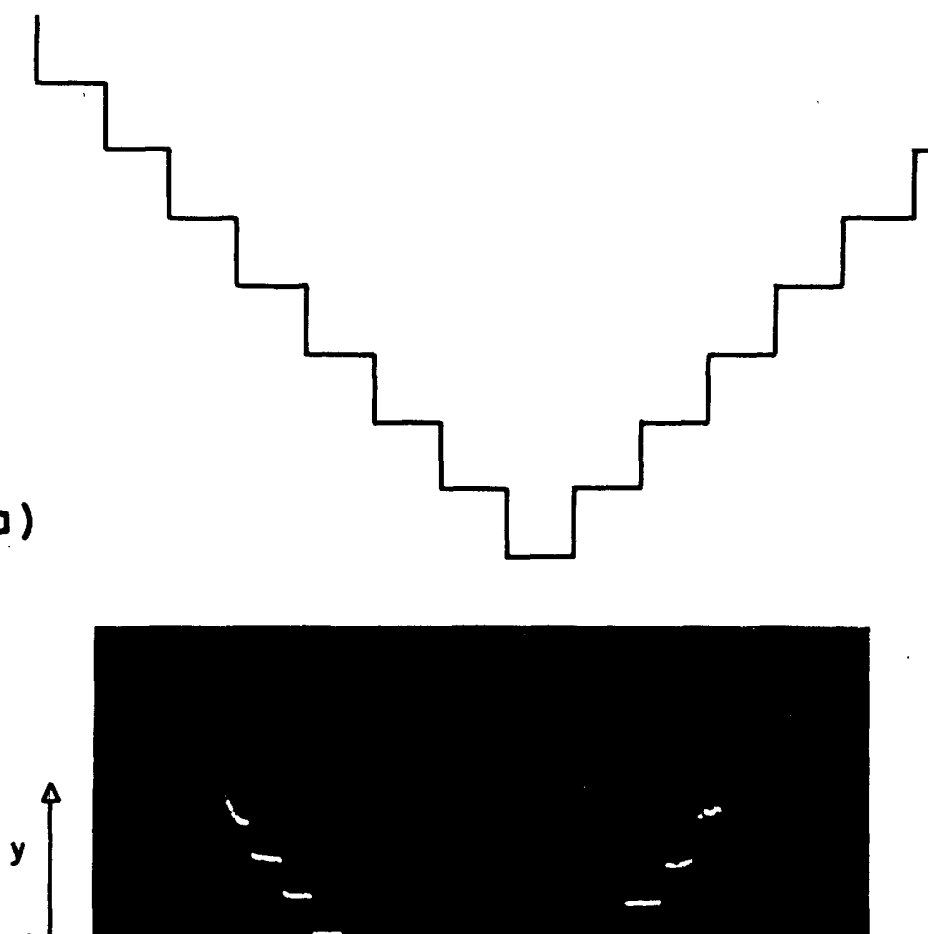

(b)

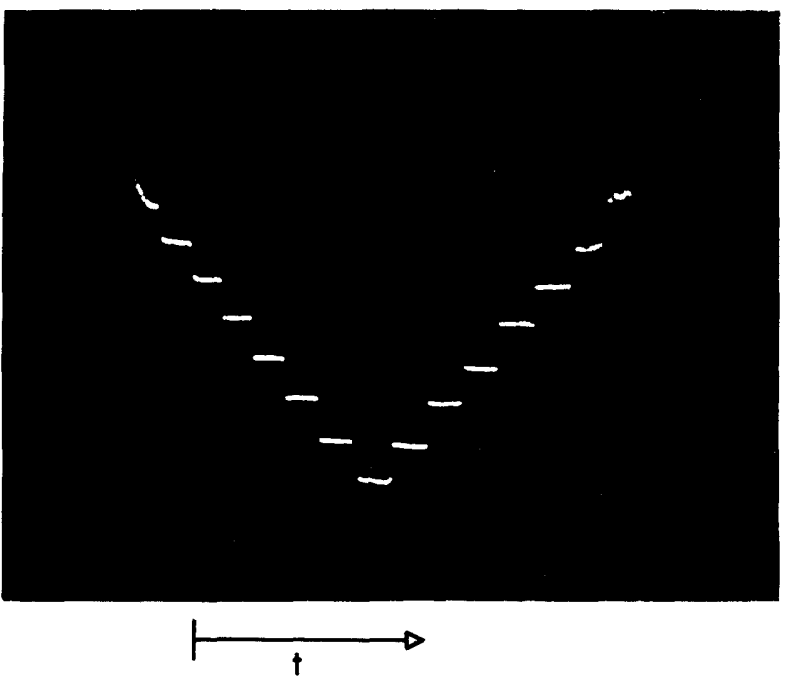

Fig.9(a) Function-generator test pattern.
Fig.9(b) Function generator generating test pattern.

* The cathode-ray tube utilized in both the crossed-fields multiplier and

P-11 screen has proved to have a sufficientiy short persistence for problems encountered to date on the electronic differential anajyzer. If a faster response were required a cathode-ray tube with the $\mathrm{P}-5$ screen might be employed. The photocelis used in the multiplier and function generator are

A number of modifications of the feedback loops employed for the crossedfields multiplier and the arbitrary function generator might be desirable in some applications ong such modification is replacement of the d-c ampilfier 1 Figures 6 and 8 by a bandpass carrier amplifier, with modulation of possibility is the replacement of the pickup photocelis in Figure 6 by a pair of collector plates mounted within the evacuated envelope of a spec1al cathode-ray tube. 


\section{G. Addition and Integration}

Electronic circuits performing the operations of addition and timeintegration are numerous and well known. The adding and integrating clrcuits employed in this electronic differential analyzer are shown in Figure 10. These basic circuits have been emplojed extensively for those specialized differential analyzers known as simulators (12)(13)(14)(15).

The circuits of Figure 10 are usually bullt around high-gain d-c coupled amplifiers which suffer from drift and warm-up problems. To avold these problems, a-c coupled amplifiers are employed in this differential analyzer. Since the repetition rate of the analyzer is $60 \mathrm{cps}$, the ampliflers must be capable of passing a $60-\mathrm{cps}$ square wave. The amount of 'droop' which can be tolerated in this square-wave response is determined by the accuracy requirements established for the differential analyzer.

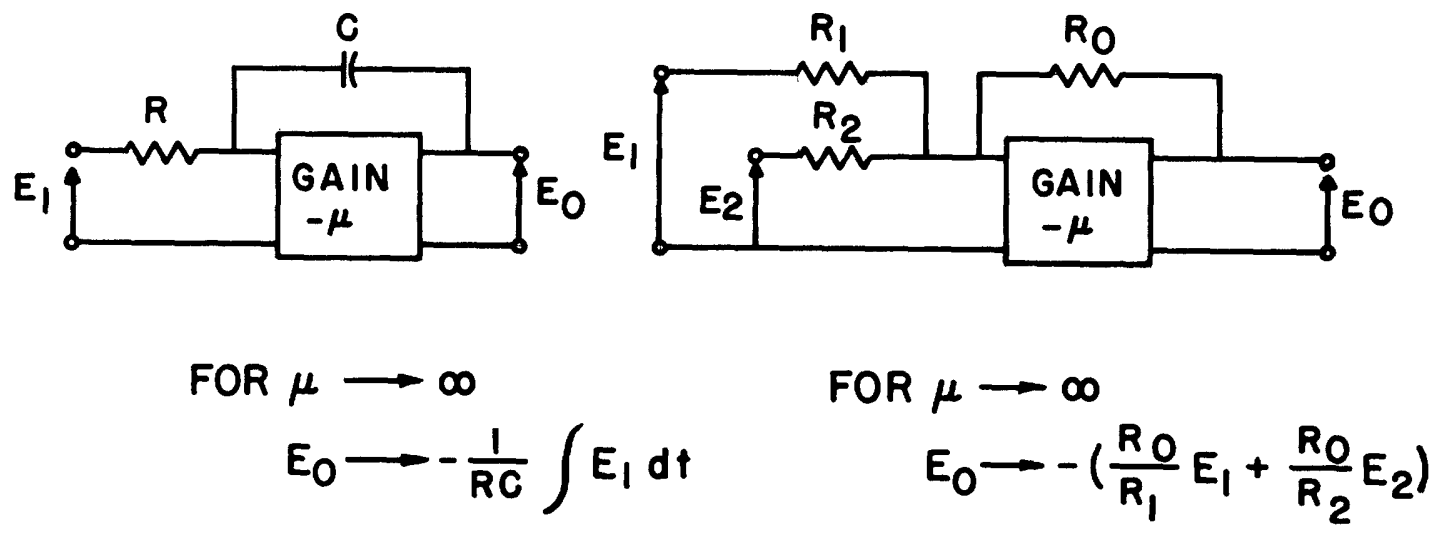

(a) INTEGRATOR

(b) ADDER

Fig.10 Integrating and adding circuito employed in the electronic differential analyzer.

\section{H. The D-C Clamping Circuit}

Because the d-c output of each computing element in the analyzer influences the initial conditions at the beginning of each solution period, means must be employed to fix the output of each computing element. This can be accomplished without losing the advantages of a-c coupling by employing some form of $d-c$ clamping; the $d-c$ clamping circuit used in the electronic differential analyzer is commonly employed to restore the d-c component in television-camera amplifiers (16)(17). This circuit is 
shown in Figure 11. A clrcult of this type is connected to each interstage coupling condenser of the adding and integrating amplifiers. The gate pulses are applied during the off-time and removed at the beginning of each on-time (see Fig.2). Although this makes the amplifiers more complicated than the usual a-c coupled amplifier, the amplifiers are still considerably simpler than d-e coupled amplifiers of comparable performance. The measured characteristics of the adding units are those listed below.

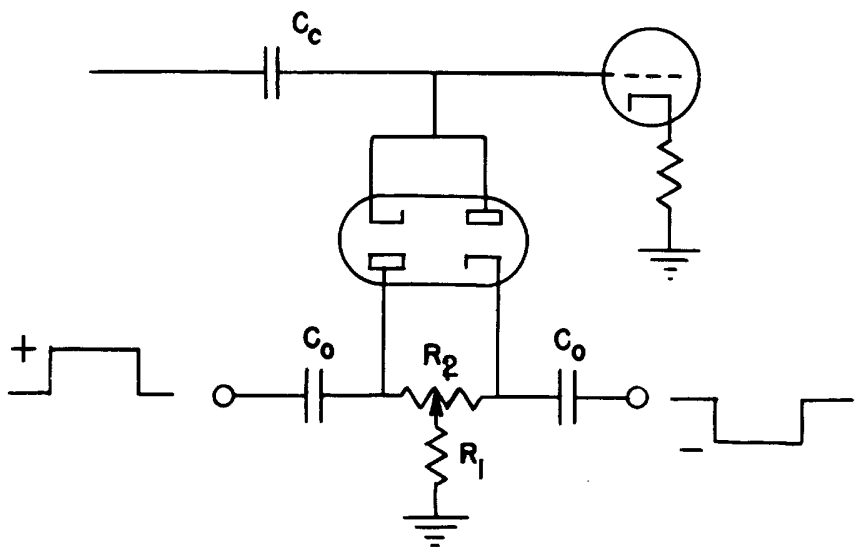

Fig.ll Pulse-controlled clamping circuit.

\author{
Measured Adder Characteristics \\ Without feedback: \\ low-frequency half-power polnt $=0.16 \mathrm{cps}$ \\ high-frequency half-power point $=100 \mathrm{kc} / \mathrm{sec}$ \\ voltage gain $=-1500$ times, $=63.5 \mathrm{db}$.
}

As used in the analyzer:

low-frequency half-power $=0.016 \mathrm{cps}$

high-frequency half-power point $=650 \mathrm{kc} / \mathrm{sec}$

rise-time (step-input $=0.4$ microseconds (10\% to $90 \%)$

overshoot $=2 \%$ or less

voltage gain $=-150$ times

output noise $=0.4$ to $5 \mathrm{mv}$

maximum output level = 120 volts

maximum output impedance $=10$ ohms

nonlinear distortion $<0.2 \%$.

I. Integrator Initial Conditions

The integrators of Figure $10(a)$ because d-c clamped amplifiers are used, automatically discharge the integrating condensers during the differential analyzer off-time. The initial condition of the integrator is set by adding a voltage step of adjustable amplitude to the output of each unit. This initial-condition step is applied at the beginning of each solution period and remains constant for the duration of the solution on-time. The inftial-condition steps can be easily and quickly adjusted by means of potentiometers, and the new solutions are obtained in $1 / 60$ second.

As indicated in Section IV, the high-and low-frequency limitations of 
the computing elements are sources of error in differential-equation solutions; a low output impedance is necessary in each computing element in order that the coaxial cables used for the interconnection of elements may not affect the element characteristics. The three important characteristics of the integrators for this electronic differential analyzer are:

1. Low-frequency time constant, at least 0.75 seconds;

2. High-frequency transient decays, to 2 per cent in 2 microseconds;

3. Output impedance, 100 ohms.

The electronic differential analyzer employing the d-c clamped adders and integrators discussed above has a warm-up time of less than five minutes. The use of d-c clamped amplifiers is advantageous for three reasons: (a) it avolds the long warm-up period and long-time instability problem of conventional d-c amplifiers; (b) it automatically turns off the analyzer and removes the final conditions from the integrator condensers at the end of every solution period, and (c) It thus permits the handing of differential equations whose solutions increase with the independent variable time. The gating and inftial-condition pulses are all generated by a single master gate generator, thus assuring synchronous operation throughout.

\section{ERRORS DUE TO COMPONENT LIMITATIONS}

Three principal types of error are encountered in the solution of differential equations by electronic means: errors caused by

1. lack of precision,

2. lack of calibration accuracy, and

3. lack of accuracy due to limitations in the time (or frequency) domain of the differential-analyzer elements.

A. Calibration Accuracy

Since the variables of this differential analyzer are voltages and time, calibrating units must be capable of measuring instantaneous voltages and times. The measurements are made on a cathode-ray tube in the present analyzer. Amplitudes are measured by direct calibration of the cathode-raytube oscilloscope; time is measured by linear oscilloscope sweeps and time pip-marker generators (18). A calibration accuracy of 2 per cent is obtained in this manner, which is adequate for the present applications.

B. Precision

Precision is of great importance in this system of solving differential equations because of the high solution-repetition rate employed. A lack of precision in setting initial conditions, for example, results in a different 
solution for the differential equation every $1 / 120$ second. Because these solutions are superimposed on the output cathode-ray-tube screen, there results a 'jitter' or fuzziness of the displayed solution.

The precision of the differential analyzer limits 1ts operation when solving equations whose solutions increase rapidly with time. This is so because the maximum output level of the analyzer components is fixed at about 25 volts peak-to-peak by the clamping circults employed. If, for example, a certain solution function increases by a factor of 2.5 during the solution time, then, for a precision of 1 per cent in the observed solution, the inftial values of the solution must be constant to 0.1 volts. If, on the other hand, a solution increases by a factor of 25 times during the solution period, then, for the same precision of solution, the initial values must be constant to within 0.01 volts. The precision of the initial condition is conveniently checked by observing a differential-equation solution which grows rapidly with time. A photograph of such a test is shown in Figure 12.

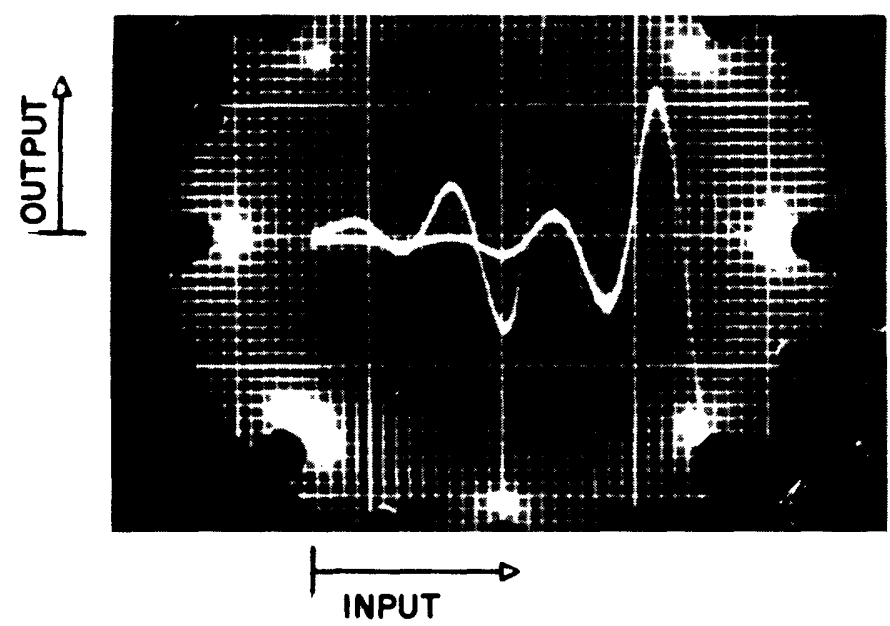

Fig.12 Precision check of differontlal analyzer.

The equation being solved in this test is

$$
\frac{a^{2} y}{d t^{2}}-2 \zeta \frac{d y}{d t}+w_{o}^{2} y=0
$$

wh1ch has the solution

$$
y=C e^{\zeta t} \cos (\omega t+\theta)
$$

where

$$
\omega_{0}>\zeta \text {, }
$$

and

$$
w=\sqrt{w_{0}^{2}-\zeta^{2}},
$$

the constants $\mathrm{C}, \theta$ are determined by the inftial conditions. 
Figure 12 is a double-exposure photograph of this sine wave with an exponentially increasing envelope. One exposure shows the entire solution; the fuzziness at the end of this trace indicates that there is about 10 per cent jitter in the initial value. The mean value of the last positive peak is 1.54 volts. The second exposure is taken with a tenfold increase in the gain of the output viewing oscilloscope. One can see that this solution increases in amplitude by a factor of 5.5 per cycle. There are 3.5 cycles of the solution from the initial value to the last positive peak; the initial value for this solution therefore, is, $1.54 /(5.5)^{3.5}=3.95$ mililvolts. Since the jitter is 10 per cent, the variability in the initial value is about 0.4 millivolts. The maximum output level for this electronic differential analyzer is 25 volts; this shows that the initial conditions are precise to within 0.002 per cent of the maximum output level. With a more usual initial value of 1 volt the precision is 0.04 per cent.

Precision of the differential analyzer is important in the solution of nonlinear equations. Exploration of the regions between stable and unstable solutions requires extreme precision. An example of such a situation is the solution of the differential equation for a physical pendulum; in stable operation the pendulum oscillates back and forth, while for unstable operation the pendulum rotates. In the critical transition case the penduIum just balances, in a position of unstable equilibrium. The degree to which one can determine this transitional case on the differential analyzer is limited by the precision of operation.

Another situation which requires utmost precision occurs in determining a particular solution of a differential equation. Such a situation is encountered, for example, in the equation

$$
\frac{d^{2} y}{d t^{2}}-4 \frac{d y}{d t}-5 y=0
$$

which has the general solution

$$
y=c_{1} e^{-t}+c_{2} e^{5 t}
$$

If it is desired to examine only the first term of this solution, it is necessary to choose those inftial values in the differential equation which make $\mathrm{C}_{2}$ zero. The precision with which this adjustment can be made limits the range over which the particular solution

$$
\mathrm{y}=\mathrm{C}_{1} \mathrm{e}^{-\mathrm{t}}
$$

can be observed, since the second, undesired, solution will, for large $t$, always mask the desired solution.

The principal factors limiting precision in the present electronic 
differential analyzer are white nolse and microphonics in the various units. The output nolse varies between 0.4 and 10 millivolts in the computing elements. By improving power supplies, shock mounting, etc., the precision could be improved by another factor of ten.

In order to obtain a good qualitative picture of the nature of the unknown solutions of a given differential equation with regard to instability, periodicity, discontinuities, etc., the important requirement on the differential analyzer is that of precision, rather than calibration accuracy. In this respect the present electronic differential analyzer is very satisfactory; for many engineering and physical applications the resulting gualitative information alone is of the greatest value.

\section{Errors Due to Frequency and Time Iimitations of Components}

The errors introduced into differential equation solutions on the electronlc differential analyzer by the time or frequency limitations of its components are of the greatest importance. Because these limitations can cause the analyzer to solve an entirely wrong equation, they have been investigated in some detall for the case of linear differential equations with constant coefficients. The results of these investigations are summarized here.

The general ordinary differential Equation (1) is solved on the electronic differential analyzer by the setup of Figure 1. The characteristic equation of this differential equation is obtained, by setting $y=e^{s t}$, as

$$
F(s)=s^{m}+\frac{A_{m-1}}{A_{m}} s^{m-1}+\ldots+\frac{A_{1}}{A_{m}} s+\frac{A_{0}}{A_{m}}=0 \text {, }
$$

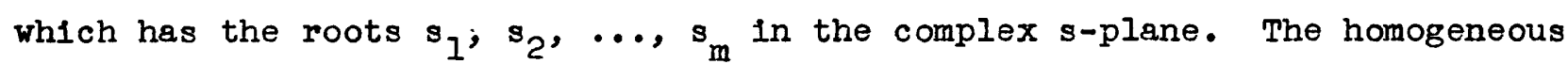
solution of Eq. (I) is of the form

$$
y=\sum_{n=1}^{m} c_{n} e^{s_{n} t}
$$

where the $C_{n}$ are constants depending upon the initial conditions of the particular solution desired.

D. Iimitation at High Frequencies

If all the components in the setup of Figure 1 are 1deal, then the solution given by Eq.(23) is observed on the differential analyzer. Ideal components are not avallable. At high frequencies the response of the addIng unit drops off because of the finite bandwidth of the amplifier employed. The response of the integrating units may also depart from the ideal at high frequencles; for this reason it is important to observe the pulse response of 
of the integrators as well as the amplifiers and adders in an electronic differential analyzer. With proper care it has been found possible always to compensate the integrator high-frequency response so as to make it better than the adding units employed*. Under this circumstance it is reasonable to calculate the errors introduced into the solution of Eq.(1) by an adder of finite bandwidth, with all other components in Figure 1 assumed ideal at high-frequencies.

If the adding unit has a $3 \mathrm{db}$ bandwidth, $\Delta f$, the differential equation solved by the setup of Figure 1 will have characteristic roots $s_{1}^{\prime}, s_{2}^{1}, \ldots, s_{m}^{\prime}$, differing slightly from the roots $s_{1}, s_{2}, \ldots, s_{m}$, plus a single new root $s^{\prime}+$. By assuming that the differences between the primed roots and the roots of Eq. (22) are small (a necessary condition if the errors are to be smal1), one can write

$$
\begin{aligned}
s_{n}^{\prime} & =s_{n}+e_{n}, \quad(n=1,2, \ldots, m) \\
& =s_{n}\left(1+\frac{e_{n}}{s_{n}}\right) .
\end{aligned}
$$

Substituting Eq.(24) in Eq.(22) and making use of the fact that $e_{n} / s_{n}<<1$ and $F\left(s_{n}\right)=0$ by definition, one can solve for the errors $e_{n}$ in root position:

$$
e_{n}=\frac{\frac{s_{n}^{2}}{2 \pi \Delta f}}{n\left[1+\frac{A_{m}-1}{A_{m}}\left(\frac{m-1}{m}\right) s_{n}^{-1}+\ldots+\frac{A_{l}}{A_{0}}\left(\frac{1}{m}\right) s_{n}^{-n+1]}\right.}
$$

for

$$
\mathrm{n}=1,2, \ldots, \mathrm{m} \text {; }
$$

and the new root $\mathrm{s}_{\mathrm{m}+1}^{\prime}$ is found to be

$$
s_{m+1}^{\prime}=-2 \pi \Delta f \text {. }
$$

Equations (25) and (26) enable one to determine the errors caused by the finite bandwidth of the adder in Figure 1 if that bandwidth, the coefficients of the differential equation, and the roots of the characteristic equation of the differential equation are known.

An experimental check on errors due to the finite bandwidths can always be made by changing the scale factor of the equation being solved. This will change the values of the equation coefficients $A_{n-1} / A_{n}$

\footnotetext{
*It will be noted that $1 t$ should be possible to build an integrator with a perfect high-frequency response, since no gain-bandwidth limitations will be violated by such a unit.
} 
and the characteristic roots $s_{n}$, but the value of $\Delta f$ will remain constant; therefore the error $e_{n}$ will change value. If no change in the character of the solution is observable when the scale-factor is changed, then the errors $e_{n}$ are negligible.

As an 1llustration, these results will be applied to the differential equation for an undamped sine wave

$$
\frac{d^{2} y}{d t^{2}}+w_{0}^{2} y=0
$$

This equation has the characteristic roots

$$
\mathbf{s}_{1}=++_{j} w_{0}
$$

and

$$
s_{2}=-j w_{0}
$$

which lie on the Imaginary axis in the complex s-plane. For an adder bandwidth of $\Delta f$, the roots of the equation actually solved are, from Eqs. (25) and $(26)$,

$$
\begin{aligned}
& s_{1}^{\prime}=\frac{\omega_{0}^{2}}{4 \pi \Delta f}+j \omega_{0} \\
& s_{2}^{\prime}=\frac{w_{0}^{2}}{4 \pi \Delta f}-j \omega_{0}, \text { and } \\
& s_{3}^{\prime}=-2 \pi \Delta f .
\end{aligned}
$$

Therefore the equation solved has the solution

$$
y=c_{0} e^{-2 \pi \Delta f t}+c_{1} e^{+\frac{\omega_{0}^{2} t}{4 \pi \Delta f}} \cos \left(\omega_{0} t+\theta\right)
$$

instead of the desired solution, which is

$$
y=c_{1} \cos \left(\omega_{0} t+\theta\right)
$$

For this example one sees that making $\Delta f$ large will cause the first term in Eq. (33) to damp out rapidly; an error in the solution will still exist, however, because the amplitude of the sine wave, instead of remaining constant, increases exponentially with time. The error from this effect is greatest at the end of the solution period $T_{0}$. If this error is to be limited to 1 per cent, then one can write as a necessary condition the inequality: 


$$
\left(\frac{w_{0}^{2}}{4 \pi \Delta f}\right) \quad T_{0}<0.01
$$

If one writes

$$
f_{0}=\frac{\omega_{0}}{2 \pi}=\text { frequency of oscillation of the solution }
$$

in cps, then solving Eq.(35) for the minimum permissible adder bandwidth gives

$$
\Delta f \geqslant 100 \pi f_{0}^{2} T_{0}
$$

For the present differential analyzer, the solution period $T_{0}$ is $1 / 120$ second (see Fig.2). If one wishes to solve Eq. (27) for $f_{0}=600 \mathrm{cps}$ (for this frequency, five complete cycles of the solution are displayed on the cathode-ray-tube screen), the adder bandwidth must be

$$
\Delta \mathrm{f}=942 \mathrm{kc} / \mathrm{sec} \text {, }
$$

if a one-per-cent amplitude increase can be tolerated. This case of the undamped sine wave imposes the most stringent conditions on the differentialanalyzer component bandwidths. (Note that this bandwidth is over 1000 times the natural frequency of the equation being solved.) For this reason the solution of Eq.(27) on the electronic differential analyzer affords a good test of one aspect of the analyzer operation. This is the same equation used to test mechanical differential analyzers in the well-known "circletest" (4).

This analysis of the influence of the adder bandwidth upon the solutions of the differential equations has been verified experimentally on the electronic differential analyzer.

\section{E. Limitation at Low Frequencies}

The principle limitation at low-frequencies is encountered in the integrating units. If the integrator of Figure 10(a) were 1deal, the Laplace transform of its gain characteristic would be

$$
\frac{e_{0}}{e_{1}}(s)=-\frac{1}{s R C}^{*} \text {. }
$$

\footnotetext{
*For real frequencies, $s=j \omega: e_{0}(\omega) / e_{1}(w)=1 \angle 90^{\circ} /(w R C)$. This emphasizes that an 1deal integrator is simply an amplifier with a constant phase shift of 90 degrees and an amplitude characteristic varying inversely with the frequency. If such on ideal amplitude characteristic a straight line having a slope of -6 db/octave. The frequencies at which an actual integrator characteristic deviates from this ideal correspond to the half-power frequencies associated with the more ordinary constant gain amplifiers and, similariy, the significant ibandwidth of an integrator is the difference between the two frequencies at which the actual characteristic differs from the ideal by $3 \mathrm{db}$.)
} 
Because of the finite amplifier gain $\mu$, the Laplace transform of the actual integrators, neglecting short-time effects, is

$$
\frac{e_{0}}{e_{1}}(s)=-\frac{L}{s R C}\left(\frac{\mu R C s}{1+\mu R C s}\right) .
$$

An analysis similar to that outlined for the high-frequency case permits one to determine that for one per cent error caused by this lowfrequency effect,

$$
\mu \mathrm{RC} \geqq \mathrm{nT}_{\mathrm{o}}
$$

where

$$
\begin{aligned}
& \mathrm{n}=\text { the order of the differential equation and } \\
& \mathrm{T}_{\mathrm{o}}=\text { the solution period. }
\end{aligned}
$$

This result has also been verifled experimentally.

The results above indicate that the effect of component limitations on the solution of ordinary differential equations with constant coefficlents on the electronic differential analyzer is subject to direct analysis. The situation for nonlinear equations and for equations with variable coefficlents is not so simple. Each equation must be treated anew and general conclusions are not easily drawn. Experimentally, one is forced to fall back on the change-of-scale-factor test outlined under high-frequency effects.

\section{RESULTS}

An electronic differential analyzer employing the general system outlined in section II has been bullt and used to solve a number of representative ordinary differential equations. Equations which have been considered in detall by analytic means were chosen so that a test of the differential analyzer operation could be obtained.

A. Solution of Simultaneous Second-Order Differential Equations

The solution of simultaneous linear differential equations with constant coefficients is of great practical importance to the engineer and physicist. Although it is possible to handle such equations directly by analytic means, a considerable amount of time and labor is required by this approach. The electronic differential analyzer affords a more economical means of obtaining such solutions.

An example of a practical electric circuit requiring the solution of a pair of simultaneous differential equations is the coupled-tuned circuit of Figure 13. For this circuit one can write the two differential equations: 


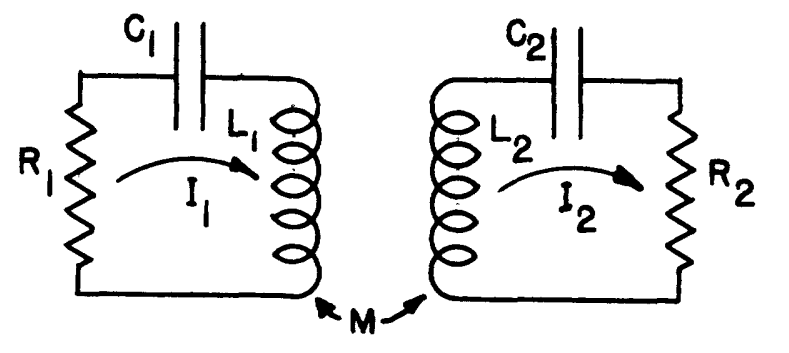

Fig.13 Coupled-tuned circuits.

$$
\begin{aligned}
& \frac{\mathrm{d}^{2} I_{1}}{d t^{2}}+\frac{R_{1}}{L_{1}} \frac{d I_{1}}{d t}+\frac{I}{L_{1} C_{1} I_{1}}-\frac{M}{L_{1}} \frac{d^{2} I_{2}}{d t^{2}}=0, \\
& \frac{d^{2} I_{2}}{d t^{2}}+\frac{R_{2}}{L_{2}} \frac{d I_{2}}{d t}+\frac{I}{L_{2} C_{2}} I_{2}-\frac{M}{L_{2}} \frac{d^{2} I_{1}}{d t^{2}}=0 .
\end{aligned}
$$

The setup for solving this pair of simultaneous differential equations on the electronic differential analyzer is given in the block diagram of Figure 14. (In this and subsequent figures, the dot notation will be used for time derivatives. Thus, for example, the symbols $\dot{I}$ and $\ddot{I}$ will denote $\mathrm{dI} / \mathrm{dt}$ and $\mathrm{d}^{2} \mathrm{I} / \mathrm{dt}^{2}$.) The solid lines in this setup indicate the connections necessary to solve elther of the differential equations individually without the mutual coupling term. Around these two loops appear all the dependent variables occuring in Eqs.(41) and (42). In order to solve these simultaneous equations, it is necessary only to interconnect these two loops as required by the mutual terms in the differential equations. These interconnections are shown as dotted lines in Figure 14. With these connections the setup of Figure 14 solves the equations

$$
\begin{aligned}
& \frac{\mathrm{d}^{2} I_{1}}{d t^{2}}=-k_{4}\left(k_{1} k_{2} I_{1}+k_{1} k_{3} \frac{d I_{1}}{d t}\right)+k_{5} \frac{d^{2} I_{2}}{d t^{2}}, \\
& \frac{d^{2} I_{2}}{d t^{2}}=-k_{9}\left(k_{6} k_{7} I_{2}+k_{6} k_{8} \frac{d I_{2}}{d t}\right)+k_{10} \frac{d^{2} I_{1}}{d t^{2}} .
\end{aligned}
$$

Comparing these equations w1th Eqs.(4I) and (42) one observes that by satisfying the relations

$$
\begin{aligned}
k_{1} k_{2} k_{4} & =\frac{1}{L_{1} C_{1}}, \\
k_{1} k_{3} k_{4} & =\frac{R_{1}}{L_{1}}, \\
k_{5} & =\frac{M}{L_{1}},
\end{aligned}
$$




$$
\begin{aligned}
k_{6} k_{7} k_{9} & =\frac{1}{L_{2} C_{2}}, \\
k_{6} k_{8} k_{9} & =\frac{R_{2}}{L_{2}}, \\
k_{10} & =\frac{M}{L_{2}},
\end{aligned}
$$

the desired solutions are obtained. There are also maximum permissible values of the integrator constants $k_{1}, k_{2}, k_{6}$, and $k_{7}$, set by the finite gain of the amplifiers as indicated in Section IV, Eq.(40). A practical procedure in setting up such a system of equations is to start by assuming that these constants have their maximum permissible values. This gives four additional constraints on the $\mathrm{k}^{\prime} \mathrm{s}$ and permits the determination of all constants uniquely from Eqs. (45) through (50). If, for one reason or another, the resulting $\mathrm{k}^{\prime} \mathrm{s}$ are elther extremely large or extremely small, a change in scale factor is usually indicated.

It will be noticed that in the arrangement of Figure 14 the integrators in the upper and lower right-hand corners have been modified slightly. With this modification, the output of the unit is the integral plus a fraction of the input as indicated. This is a simple scheme to save an amplifying unit, as shown in Figure 15. Both circults of Figure 15 form the integral of the input plus a fraction of the input. The circuit to the left has the advantage of requiring one less amplifier. It has the disadvantage that changing $\mathrm{k}_{2}$ independently of the other constants requires changing the resistor $R_{2}$, which is inside a feedback loop. In the second circult, all constants can be varied by potentiometers at the input terminals of the various units without disturbing the feedback loops. For the higher-order differential equations, where the number of computing units required may become very high, the circuit at the left and similar devices are very useful.

Some typical solutions of Eqs.(41) and (42) as observed on the electronic differential analyzer are shown in Figures 16 and 17 . Figure 16 is a double-exposure photograph showing the primary and secondary currents as a function of time for the nondissipative case $\left(R_{1}=R_{2}=0\right)$. The 1lluminated scale in the photograph is ruled on an illuminated graticule placed over the output cathode-ray-tube screen. Figure 17 is another double-exposure record of the primary and secondary currents versus time in the more general case of dissipation in both the primary and secondary.

It is clear that these solutions of two simultaneous differential equations are equivalent to the solution of a single fourth-order differential equation. Differential equations of orders higher than the sixth have 


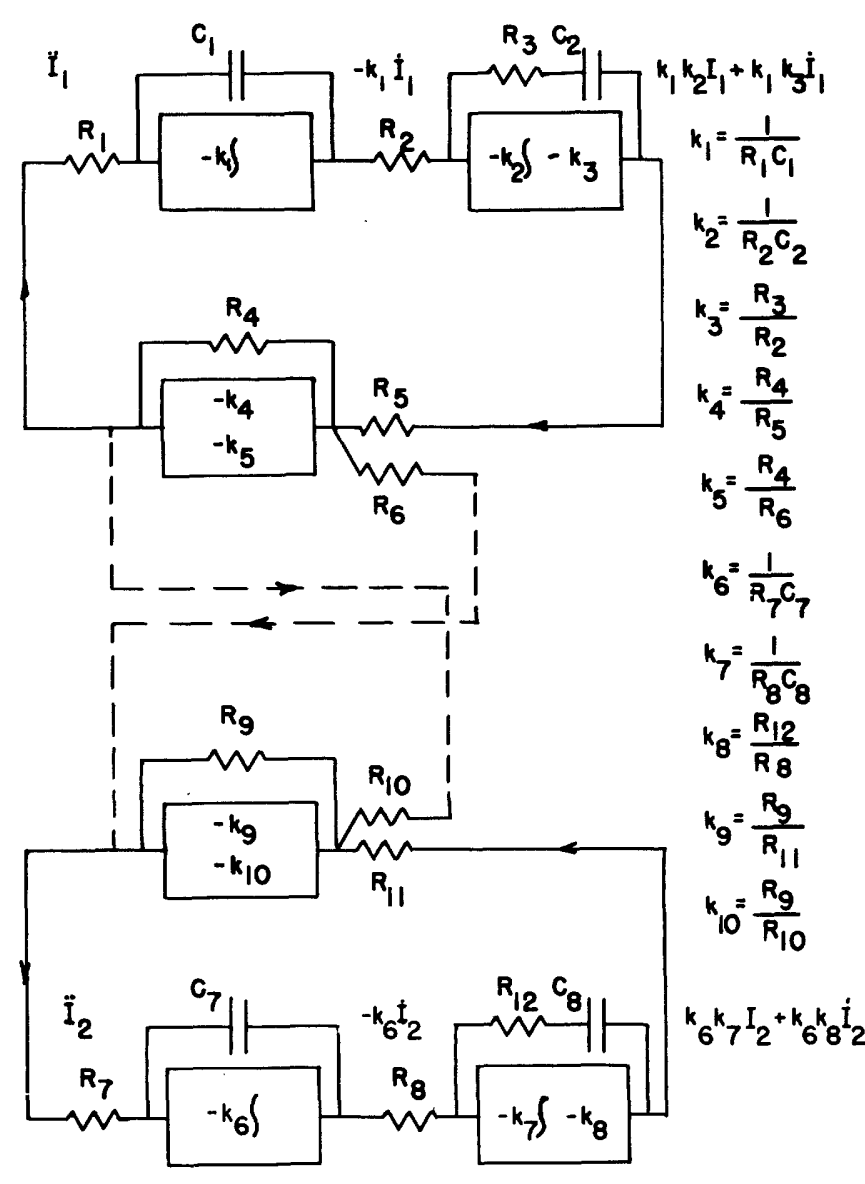

Fig.14 Differential-analyzer setup for solving simultaneous secondorder differential equations.

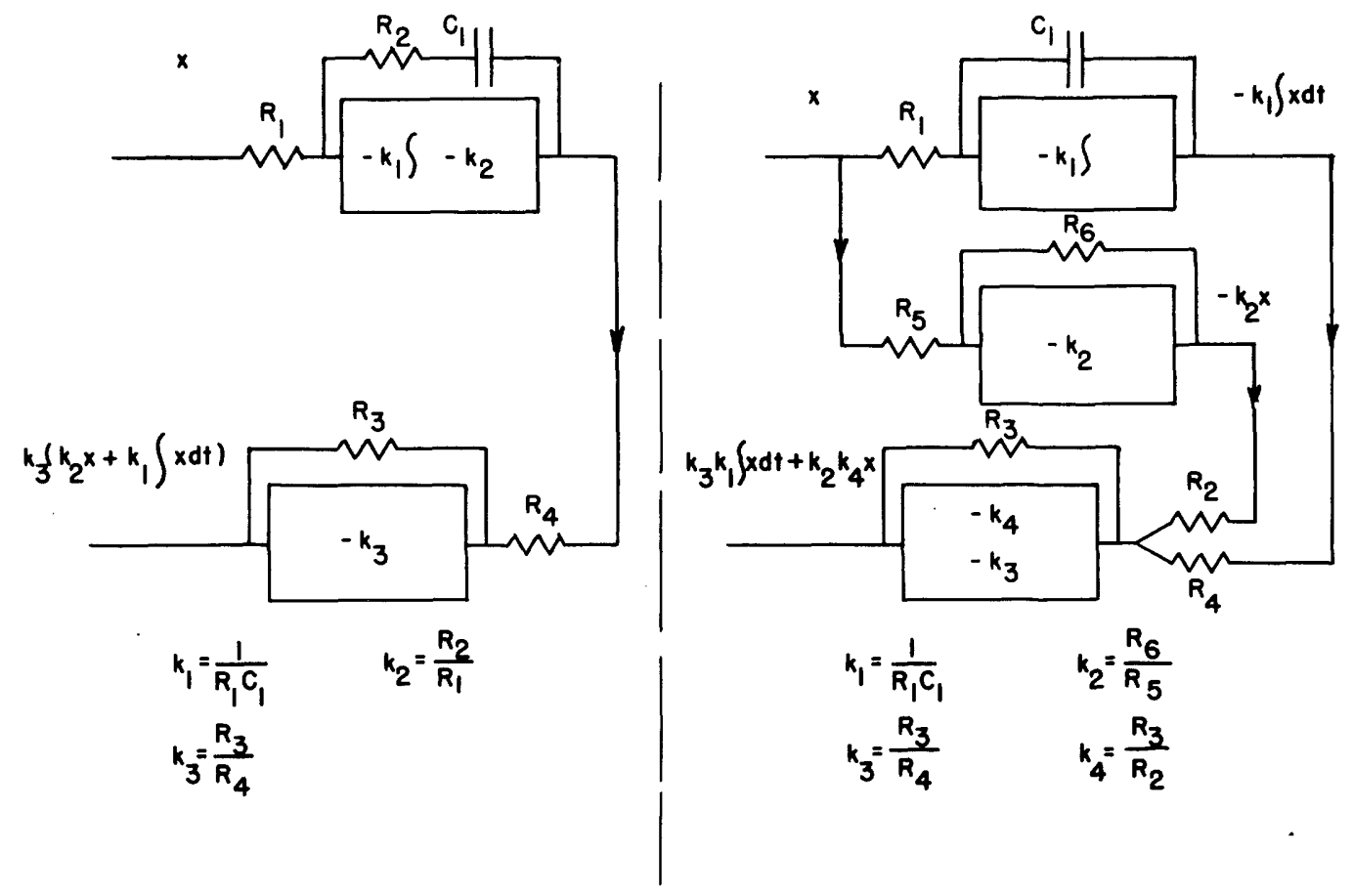

Fig.15 Two equivalent computing circuits. 


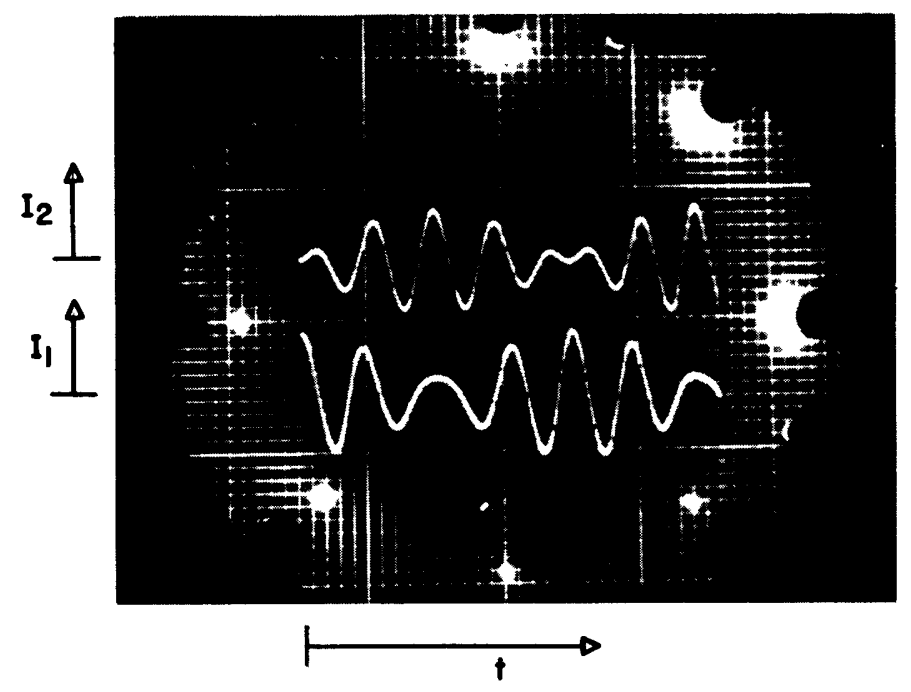

F1g.16 Primary and secondary currents versus time, for nond 1ss1pative coupled circuits.

$\dot{I}_{10}=I_{20}=\dot{I}_{20}=0, I_{10} \neq 0$.

F1g.17 Primary and secondary currents versus time, for coupled circuit withdissipation. $\dot{I}_{10}=I_{20}=\dot{I}_{20}=0, I_{10} \neq 0$.

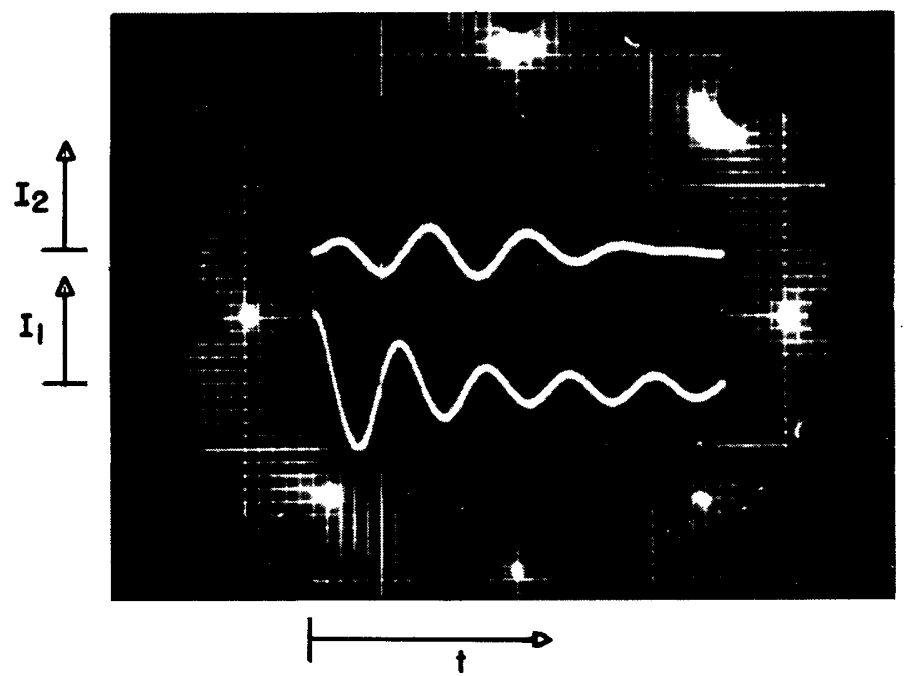

not deen solved on the present model of the electronic differential analyzer because only six integrating units have so far been built. Since no unusual difficulties have been encountered in solving equations of fifth and sixth order, it is felt that the extension of the electronic differential analyzer to the solution of equations of even higher orders should not present any insurmountable difficulties.

\section{B. Solution of Time-Varying Force Equation}

An Interesting group of Ilnear equations with variable coefficients, not easily treated by analytic methods, have the form

$$
\frac{d^{2} y}{d t^{2}}+F(t) y=0
$$


$F(t)$ is called the "force function".

The differential-analyzer setup for the solution of such equations is given in Figure 18. The multiplier shown in this figure is the crossedfields multiplier discussed in Section III; the amplifier of gain $-k_{4}$ inserted in the setup is a convenience; its use permits an adjustment in scale factors to assure that the multiplying unit always operates over its most favorable range.

\section{The Mathieu and Hill Equations}

If one chooses

$$
F(t)=\omega_{0}^{2}\left(I+\epsilon \cos \omega_{m} t\right)
$$

In Eq.(5I), one obtains the well-known Mathieu equation(Ref.19). This equation is encountered in the solution of Laplace's equation in elliptical coordinates and in numerous other problems of physics and engineering. One such problem of considerable interest to electrical engineers is discussed below.

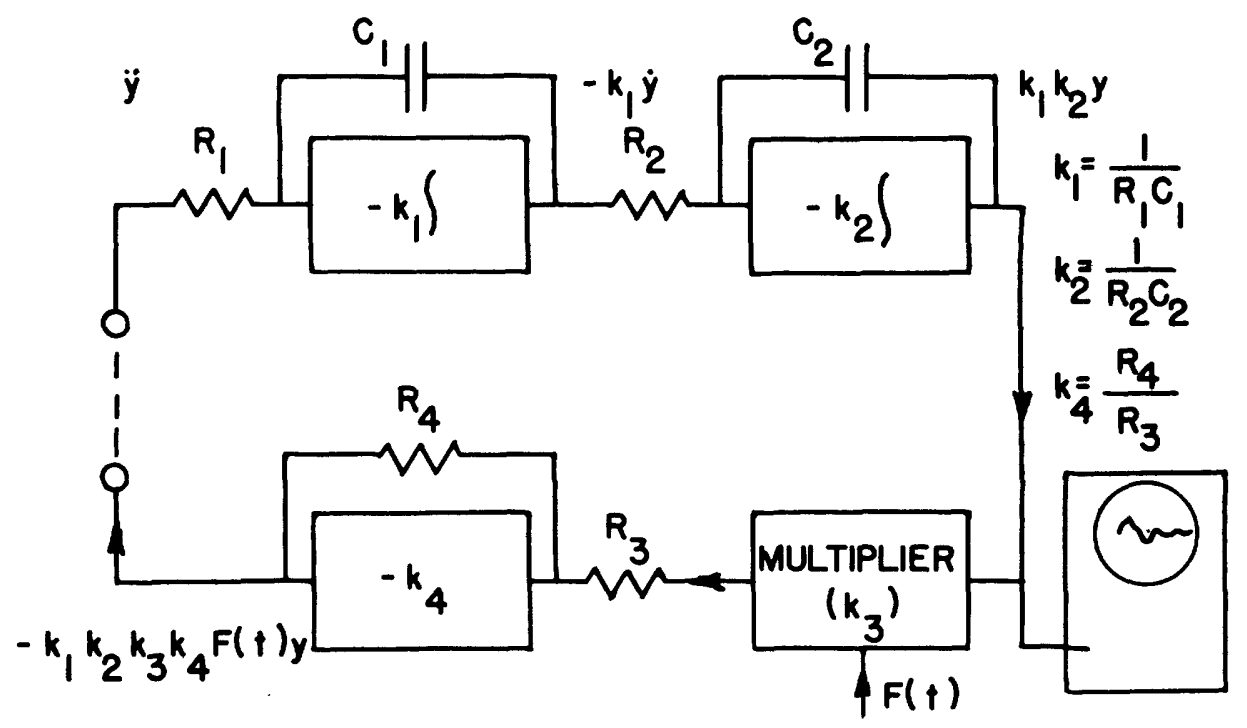

F1g. 18 Differential-analyzer setup for the solution of the equation $\ddot{\mathrm{y}}+F(t) \bar{y}=0$.

If for the nondissipative clrcult of Figure 19 the capacity is made to vary according to the relation

$$
C=C_{0}\left(1-\epsilon \cos \omega_{m} t\right),
$$

one obtains for the differential equation describing the behavior of the current in this circuit the relation: 


$$
\frac{d^{2} I}{d t^{2}}+\frac{I}{L_{0} C_{0}\left(I-\epsilon \cos \omega_{m} t\right)}=0 \text {. }
$$

This physical situation has been of considerable interest in connection with the generation of acoustic warble tones since the time of Helmholtz and Raylelgh and, more recently, has been studied in connection with frequency modulation $(20)$. If $\epsilon \ll 1$ and $\omega_{m}<<\omega_{0}$ (where $\omega_{0}^{2}=1 / L_{0} C_{0}$ ), Eq. (54) corresponds to a typlcal frequency-modulated broadcast oscillator, as commerclally employed.

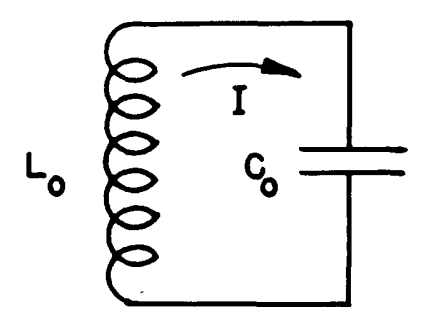

Fig.19 Parallel LC circuit.

Equation (54), without the assumptions $\epsilon \ll 1$ and $w_{m} \ll \omega_{0}$, is of the Hill type and is very difficult to handle analytically. Barrow, in studyIng this problem, made the assumption that $\epsilon \ll I$ (2I). This enabled him to use the approximation

$$
\frac{1}{1-\epsilon \cos \omega_{m} t} \approx 1+\epsilon \cos \omega_{m} t .
$$

Substituting Eq.(55) in Eq.(54), one obtains

$$
\frac{d^{2} I}{d t^{2}}+w_{0}^{2}\left(I+\epsilon \cos \omega_{m} t\right) I=0,
$$

which is the Mathieu equation. Both this equation and the original Eq.(54) of the H1ll type can be handled with equal ease on the electronic differentlal analyzer, although analytically Barrow found it necessary to change immediately to the Mathleu equation from the original Hill equation because of the "formidable mathematical difficulties" of the general case. Cambl has recently studied the original Hill equation (Ref.22).

The Mathieu equation is solved on the electronic differential analyzer with the setup of Figure 18. The function $F(t)$ given by Eq.(52) is conveniently generated by solving the auxiliary differential equation

$$
\frac{d^{2} x}{d t^{2}}+w_{m}^{2} x=0,
$$

and adding a constant voltage to the solution. This auxiliary equation is 


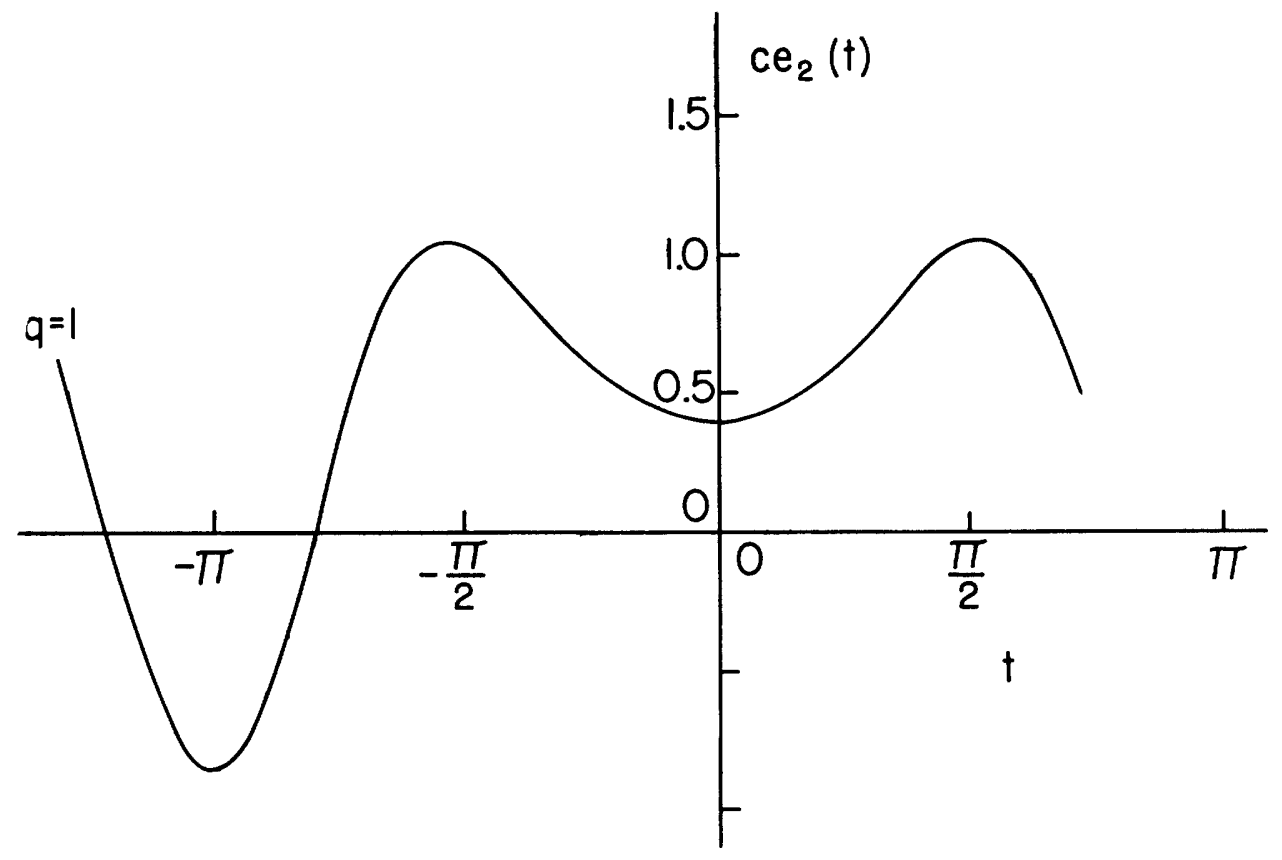

Fig.22 Calculated Mathieu function, $\mathrm{ce}_{2}(t)$.

The new setup is shown in Figure 23. Division is performed as shown in Figure 23 by employing the crossed-fields multiplier as an element in the feedback loop of an adding amplifier (15). Comparing the two setups of Figures 18 and 23 one sees that by moving one connection and adding one connection it is possible to shift the differential-analyzer setup from the Mathieu to the Hill equation, a simple operation, whereas the difficulty of the corresponding analytic change is enormous. These analytic problems are so great as to have prevented any considerable use of equations of this level in normal engineering work. With the avallablitty of the electronic differential analyzer, this situation no longer need exist.

A typical solution of the Hill equation is shown in Figure 24. This is a triple-exposure photograph showing the current and its first and second derivatives as functions of time for the case of $\epsilon \approx 0.5$ and $w_{0} / w_{m}=4$.

Figure 25 is a triple-exposure photograph of solutions of the Mathieu equation for the same values of $\epsilon=0.5$ and $w_{0} / w_{m}=4$. Comparing Figures 24 and 25 one sees that for these conditions the approximation of Eq. (55) is certainly invalid.

\section{E. Nonlinear Differential Equations}

The linear equation

$$
\frac{d^{2} y}{d t^{2}}-A \frac{d y}{d t}+y=0
$$

has the solution: 
solved by the arrangement of Figure 20. Thus Figures 18 and 20 together comprise the complete setup for the solution of the Mathieu equation on the electronic differential analyzer.

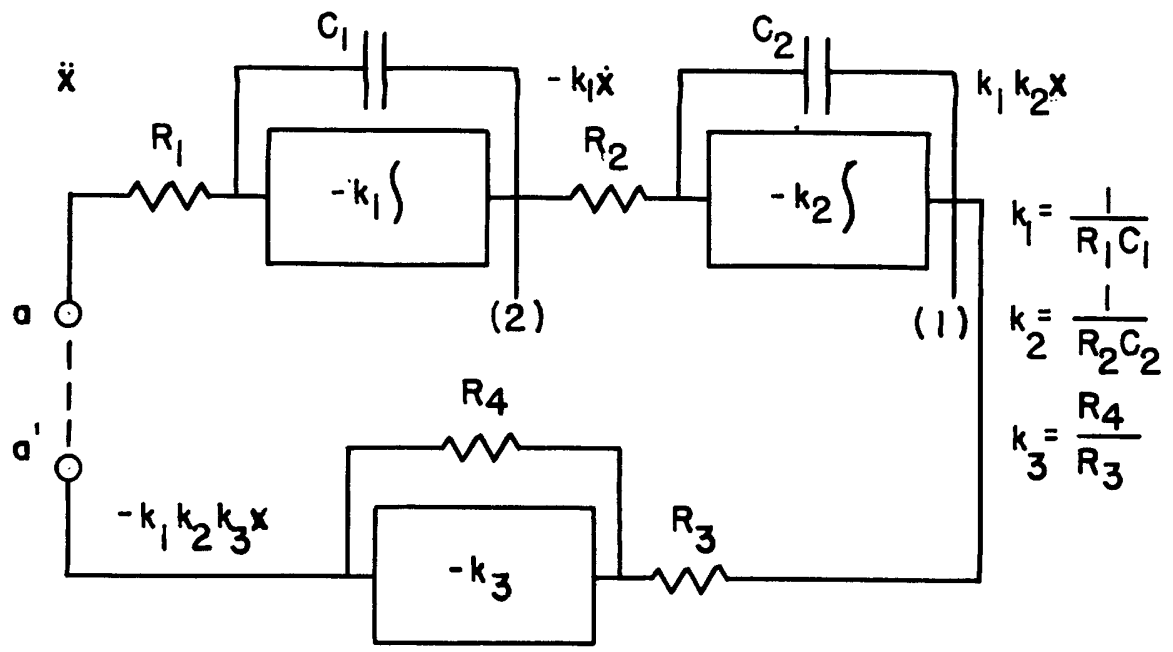

Fig.20 Differential-analyzer setup for the solution of the equation $\ddot{x}+w_{m}^{2} x=0$.

The Mathieu functions are special solutions of the Mathieu equation which are periodic in behavior (19). Figure 21 shows the differential-analyzer generation of the Mathieu function $c e_{2}(t)$. Figure 22 shows the calculated solution for this case as taken from Jahnke and Emde (23).

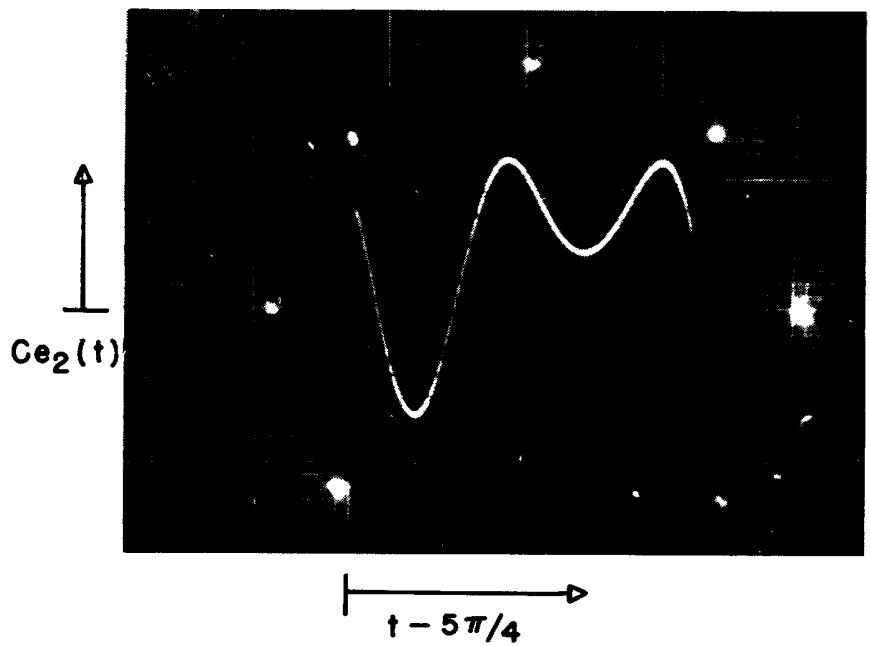

Fig.21 Mathieu function, $c e_{2}(t)$, as observed on the differential analyzer.

D. The Hill Equation

Solving the analytically more-difficult Hill equation requires only that the multiplier in the setup of Figure 18 be replaced by a divider. 


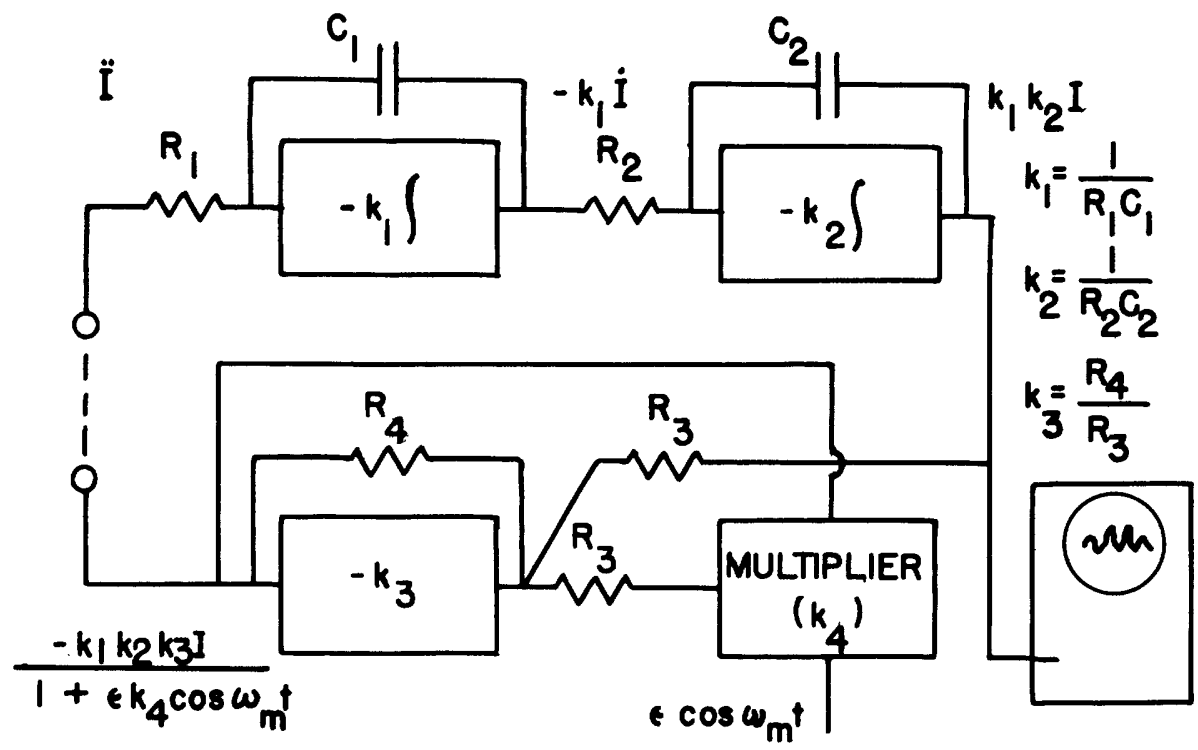

Fig.23 Differential-analyzer setup for the solution of the Hill equation.
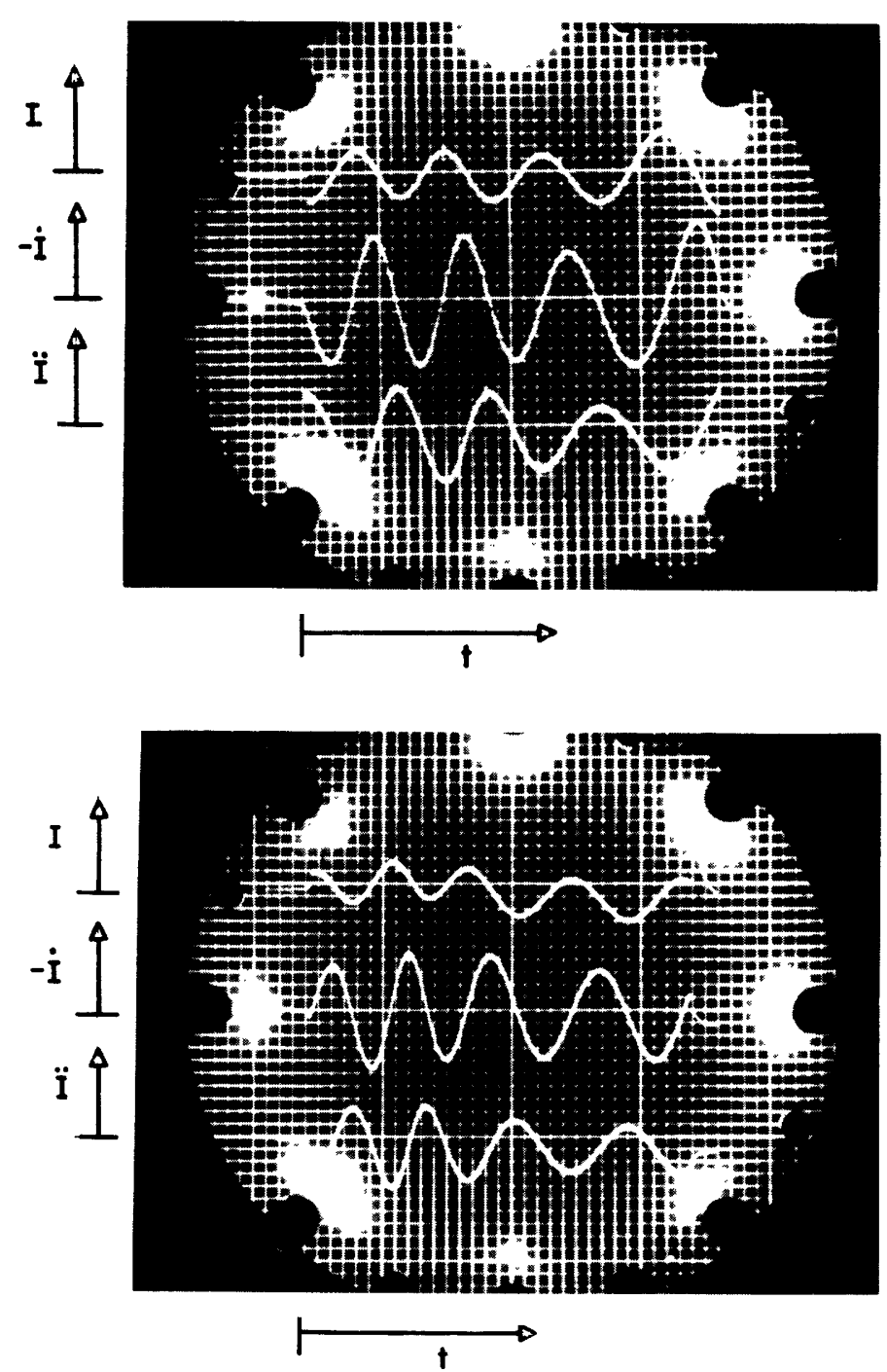

Fig.24 Solution of the Hill equation, $\frac{d^{2} I}{d t^{2}}+\frac{\omega_{0}^{2} I}{1+\epsilon \cos \omega_{m} t}=0$ for $\epsilon=0.5$ showing $I,-\dot{I}$, and $\ddot{I}$ versus $t$.
F1g.25 Solution of the Mathieu equation, $\frac{d^{2} I}{d t^{2}}+\left(1+\epsilon \cos \omega_{m} t\right)$ $\omega_{0}^{2} I=0$, for $\epsilon=0.5$ and $\omega_{0} / \omega_{m}=4$ showing $I,-\dot{I}$, and $\ddot{I}$ versus $t$. 


$$
y=c_{0} e^{A t / 2} \cos \left[\left(I-\frac{A^{2}}{4}\right)^{I / 2} t+\theta\right]
$$

where $C_{0}$ and $\theta$ are constants depending upon the initial conditions, and $A^{2} / 4<1$. Equation $(58)$ is applicable to many practical self-excited oscillators for small amplitudes of oscillation. Thus Eq.(59) describes, for example, the manner in which oscillation begins to build up in a selfexcited vacuum-tube oscillator. Because this equation is inear, it can provide no information concerning the ultimate amplitude to which the oscillation builds up, or the steady-state waveform of the oscillation. In order that these most important characteristics be studied, it is necessary to take account of the nonlinearities which are inevitable in any physical oscillator.

The well-known Van der Pol equation (Ref.24),

$$
\frac{d^{2} y}{d t^{2}}-\left(A-3 B y^{2}\right) \frac{d y}{d t}+y=0 \text {, }
$$

is an extensively studied nonlinear equation pertaining to many types of oscillations. This equation describes a system in which the damping is negative for small amplitudes of oscillation and positive for large amplitudes of oscillation.

Solution of the Van der Pol Equation (60) on the electronic differential analyzer requires the use of a function generator to generate $y^{2}$ from $y$ and a multiplier to form the product, $y^{2} \frac{d y}{d t}$. From a practical point of view it is desirable to keep the number of multipliers and function generators required by the differential analyzer setup to a minimum, because these are the most complicated units of this electronic differential analyzer. It is worth while, therefore, to consider if Eq.(60) can be simplified for machine solution by a change of variable. Such a change of variable can, in fact, be made; namely

$$
x=\int y d t .
$$

Applying this to Eq. (60) one obtains

$$
\frac{d^{3} x}{d t^{3}}-A \frac{d^{2} x}{d t^{2}}+3 B \frac{d x}{d t} \frac{d^{2} x}{d t^{2}}+\frac{d x}{d t}=0,
$$

which can be integrated term-by-term, with respect to time, to give

$$
\frac{d^{2} x}{d t^{2}}-\left[A-B\left(\frac{d x}{d t}\right)^{2}\right] \frac{d x}{d t}+x=0 \text {. }
$$


This equation, discovered by Lord Rayleigh in connection with acoustic phenomena, is known as Rayle1gh's equation(Ref.24). On writing this equation in the form

$$
\frac{d^{2} x}{d t^{2}}=-x+A \frac{d x}{d t}-B\left(\frac{d x}{d t}\right)^{3},
$$

the block diagram of the differential-analyzer setup can easily be determined, as shown in Figure 26. The advantage of solving the Raylelgh equation lies in the fact that there is required only a single function generator to generate the cube of the first derivative instead of a function generator plus a multiplier.

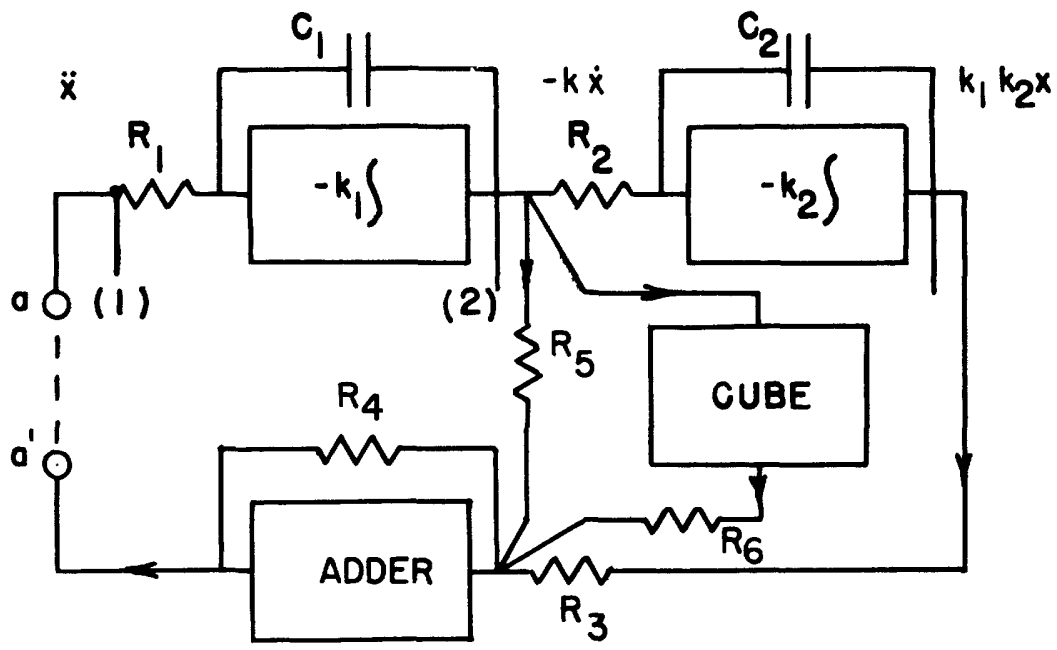

Fig. 26 Block diagram of the differential-analyzer setup for the solution of Rayleigh Equation (63).

F. Typical Solutions

Rayleigh's equation has been solved on the electronic differential analyzer using the arbitrary function generator described in section III. If the first derivative is displayed as a function of time, according to Eq.(61), the solution of the Van der Pol equation is observed. A typical solution of the Van der Pol equation, as observed on the electronic differential analyzer, is shown in Figure 27. The amplitude envelope starts to build up exponentially; in a short time, however, the rate of amplitude rise drops and the amplitude approaches a constant value because of the nonlinear damping term. The case shown corresponds to what is normally referred to as the high-Q case in electrical engineering problems. It is the situation in which the energy increase per oscillation is small compared to the peak stored energy during the build up period; this means that a large number of 
oscillations occur during the build-up period. Mathematically, the high$Q$ case indicates that both $A$ and $B$ in Eq. (63) are small compared to unity. Another solution display, which is of great interest to the mathematiclan and englneer, is the "phase-space" plot, which is a plot of velocity versus displacement (24). Such a plot is easily obtained on the electronic differential analyzer by connecting the vertical deflecting system of the viewing oscilloscope to Point (1) and the horlzontal input to Point (2) of Figure 26. A phase-space plot for the Van der Pol equation is shown in Figure 28. The build-up to the steady-state "limit cycle" is clearly shown by this photograph. For the engineer, the shape of this limit cycle is useful in determining the steady-state waveform of the oscillator. If the steady-state osclilation were exactly sinusoldal, the limit cycle would be exactly c1rcular.

To obtain the low $-Q$ solution, in which the oscillation very rapidy reaches its steady state, it is necessary merely to increase the adder gain on the inputs connected through $R_{5}$ and $R_{6}$ shown in Figure 26. By decreasing the gain at $R_{3}$ at the same time, the number of solution cycles of the display is reduced, so that the detalls of the initial rapid bulld-up for this case can be clearly seen. A typical low $Q$ solution is plotted versus time In Figure 29. The corresponding phase-space plot, with a now-much-distorted limit cycle, is shown in Figure 30.

It should be emphasized that the time necessary to shift between these two widely different solutions of Figures 27 and 29 on the electronic differential analyzer is merely the time necessary to adjust two or three knobs. One can, for example, explore in a very short perlod the solutions existing for the entire range between the two cases shown. If no record of the solutions is made, such an exploration takes the operator a matter of minutes; if photographic records are required, it is possible to obtain such recorded solutions at the rate of two or three per minute.

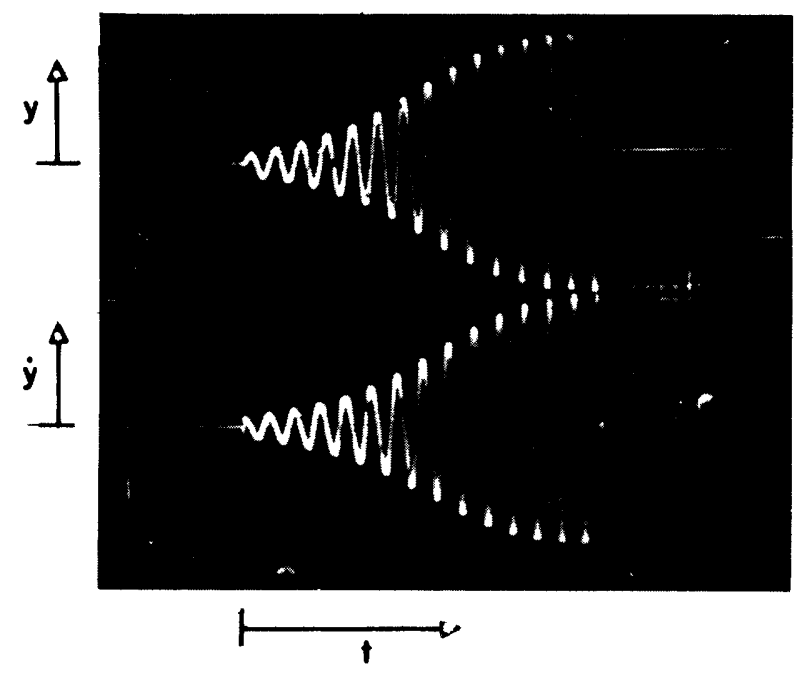

Fig.27 Solution of Van der Pol equation for the high-Q case 8 observed on the electronic differential analyzer. This is a double-exposure photograph showing $y$ and $\dot{y}$ as functions of time. 

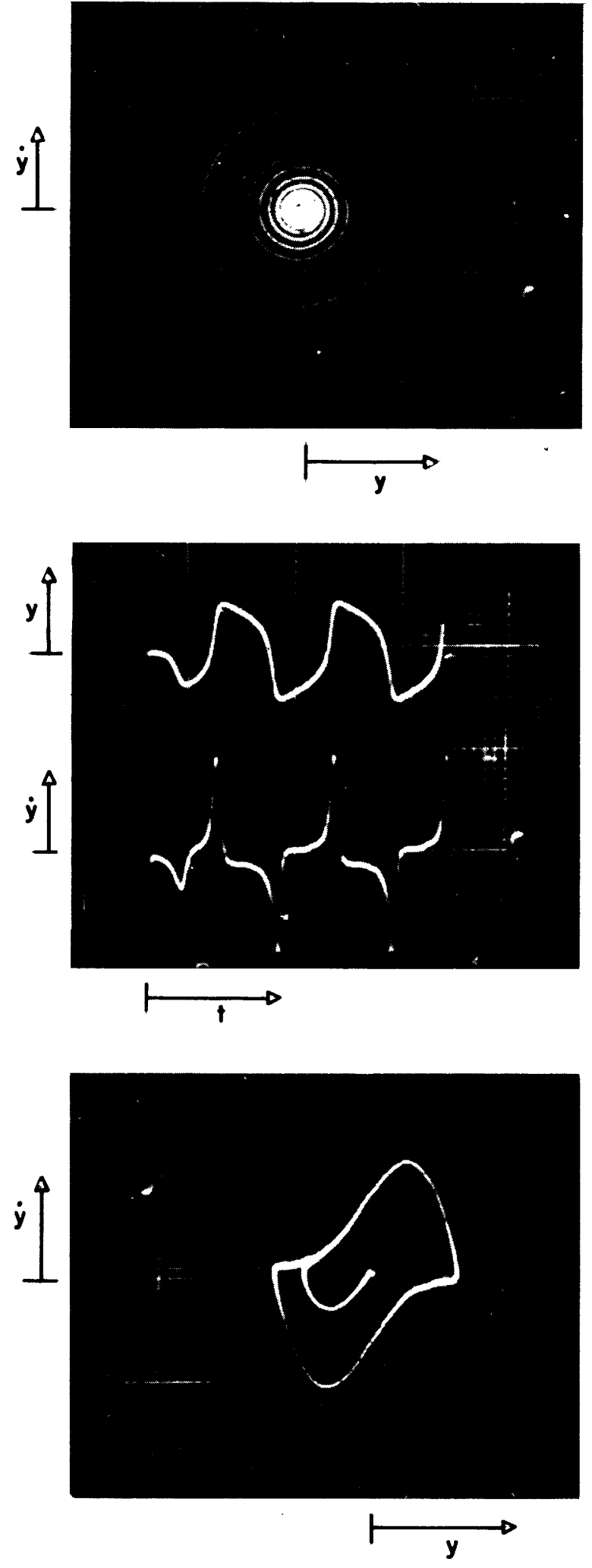

F1g.28 Phase-space plot for the Van der Pol equation, high-Q case.
Fig.29 Solution of the Van der Pol equation for the low $-Q$ case. This is a double-exposure photograph showing $y$ and $\dot{y}$ as functions of time.
Fig.30 Phase-space plot for Van der Pol equation, low $-Q$ case. 
G. Solution of an End-Point Boundary-Condition Problem

As was indicated in the introduction, a most important result of the high-speed operation of the electronic differential analyzer is the ability to handle differential equations with unspecified initial conditions. An example of such a problem is to find the solution of the equation

$$
\frac{d^{3} y}{d t^{3}}-\frac{y}{4}\left(t-t_{0}\right)=0
$$

over the range $0<t<t_{0}$, subject to the condition that at

$$
\begin{aligned}
& t=t_{0}=\frac{5 \pi}{2}, y=-1.84 \\
& \frac{d y}{d t}=0,
\end{aligned}
$$

and

$$
\frac{d^{2} y}{d t^{2}}=+0.64
$$

In the operating differential analyzer, the independent variable, time, always runs from zero to $T$ seconds, where $T$ is usually about $1 / 120$ second, as indicated in Figure 2. Therefore, in order to solve Eq. (65), it is first necessary to make the change of variable

$$
t^{\prime}=t \frac{T}{\frac{5 \pi}{2}}=n t \text {. }
$$

Then Eq. (65) becomes

$$
\frac{d^{3} y}{d t^{\prime 3}}=\frac{y}{4 n^{4}}\left(t^{\prime}-t_{0}^{\prime}\right),
$$

which is to be solved over the range $0 \ll t^{\prime} \ll T$, with the final conditions at $t^{\prime}=T$ of

$$
\begin{aligned}
y & =-1.84 \\
\frac{d y}{d t^{\prime}} & =-0 \\
\frac{d^{2} y}{d t^{\prime 2}} & =\frac{0.64}{n^{2}} .
\end{aligned}
$$

The setup necessary to solve Eq.(68), subject to the final conditions of Eq.(69), is given in Figure 31. Four integrators and the crossed-fields multiplier are required. If the integrator constants $k_{1}, k_{2}$, and $k_{3}$ are chosen equal to $1 / n$, one can observe with a viewing oscilloscope the ratio of $\mathrm{y} / \mathrm{n}^{2} \dot{\mathrm{y}}$, which, according to Eq. (69), must equal -2.88 at $\mathrm{t}^{\prime}=\mathrm{T}$. 
Figure 32 gives solutions to Eq. (68) for $i_{0}^{\prime}=T=1 / 100$ second. By observing the initial values of these curves, one finds that to satisfy the final conditions of $\mathrm{Eq} .(69)$ the initial conditions must be

$$
\begin{aligned}
& \left.y\right|_{t=0}=-0.05, \\
& \left.\frac{d y}{d t^{\prime}}\right|_{t=0}=0, \\
& \left.\frac{d^{2} y}{d t^{\prime 2}}\right|_{t=0}=-\frac{0.03}{n^{2}} .
\end{aligned}
$$

The solutions of Figure 32 were obtained after about an hour of trialand-error investigations, varying the unknown initial value. During this exploratory period, about 200 trial solutions were run; the same number of trial solutions on one of the slower mechanical differential analyzers would, conservatively, require about three elght-hour working days. A calculated solution for Eq.63 is shown in Figure 33 .

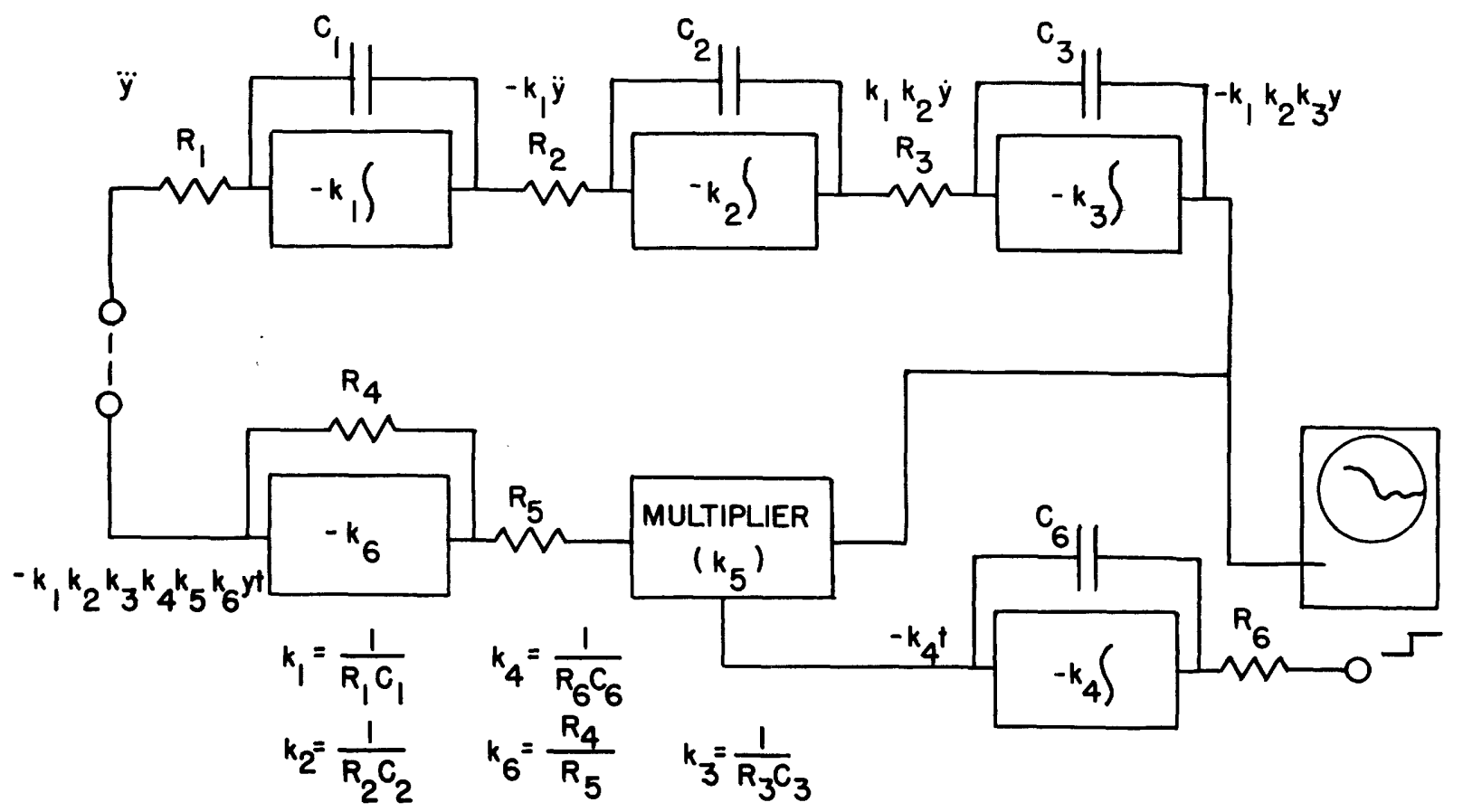

Fig. 31 Differential-analyzer setup for solving the differential equation $d^{3} y / d t^{3}=y\left(t \cdot-t_{0}{ }_{0}\right) / 4 n^{4}$. 

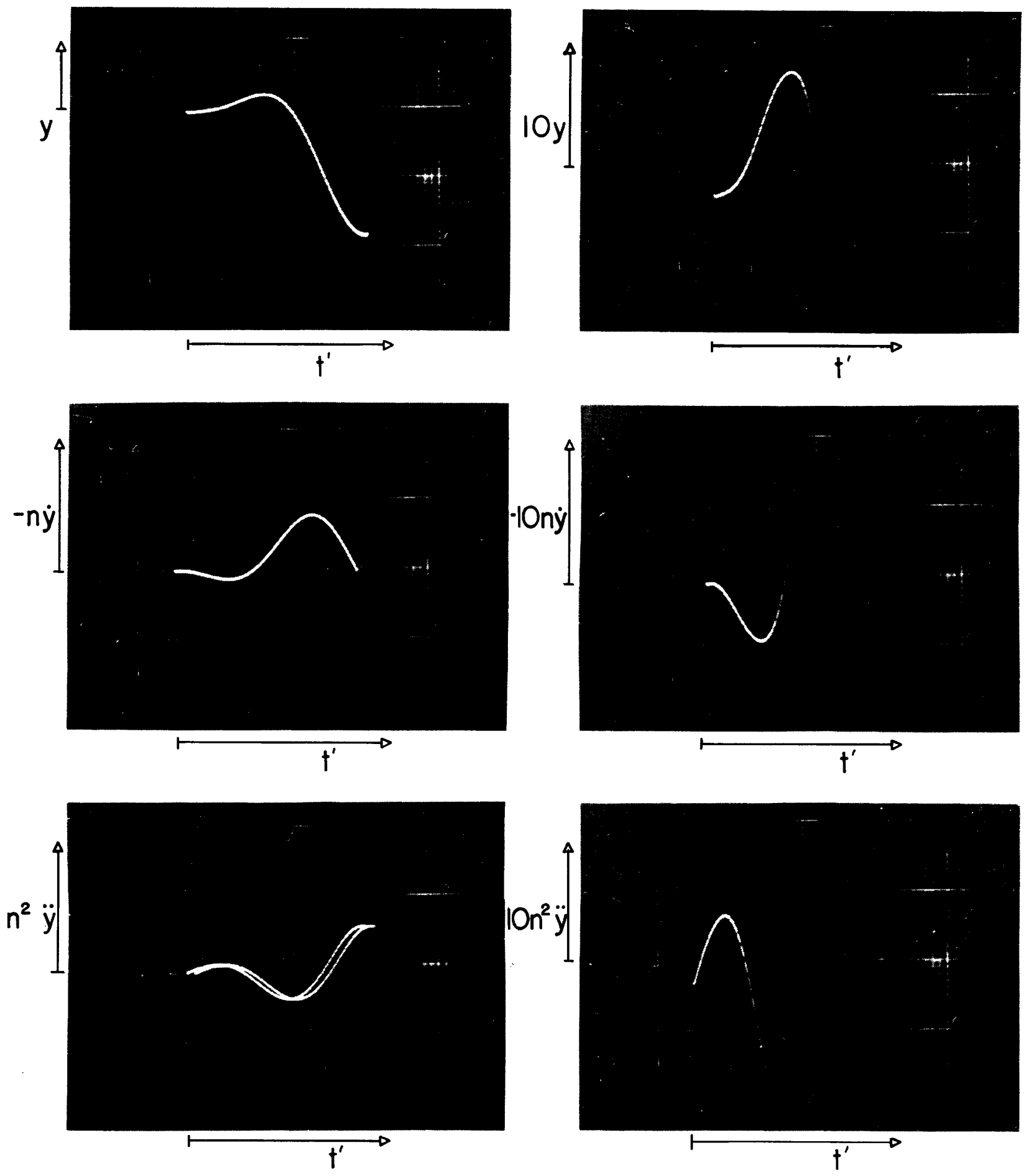

Fig.32 Solution of the differential equation $\dddot{y}=y\left(t^{\prime}-t_{0}^{\prime}\right) / 4 n^{4}$ for $0 \gtrless t^{\prime} \ll t_{0}^{\prime}$; plots of $y,-n \dot{y}$ and $n^{2} \ddot{y}$ as functions of $t$. 


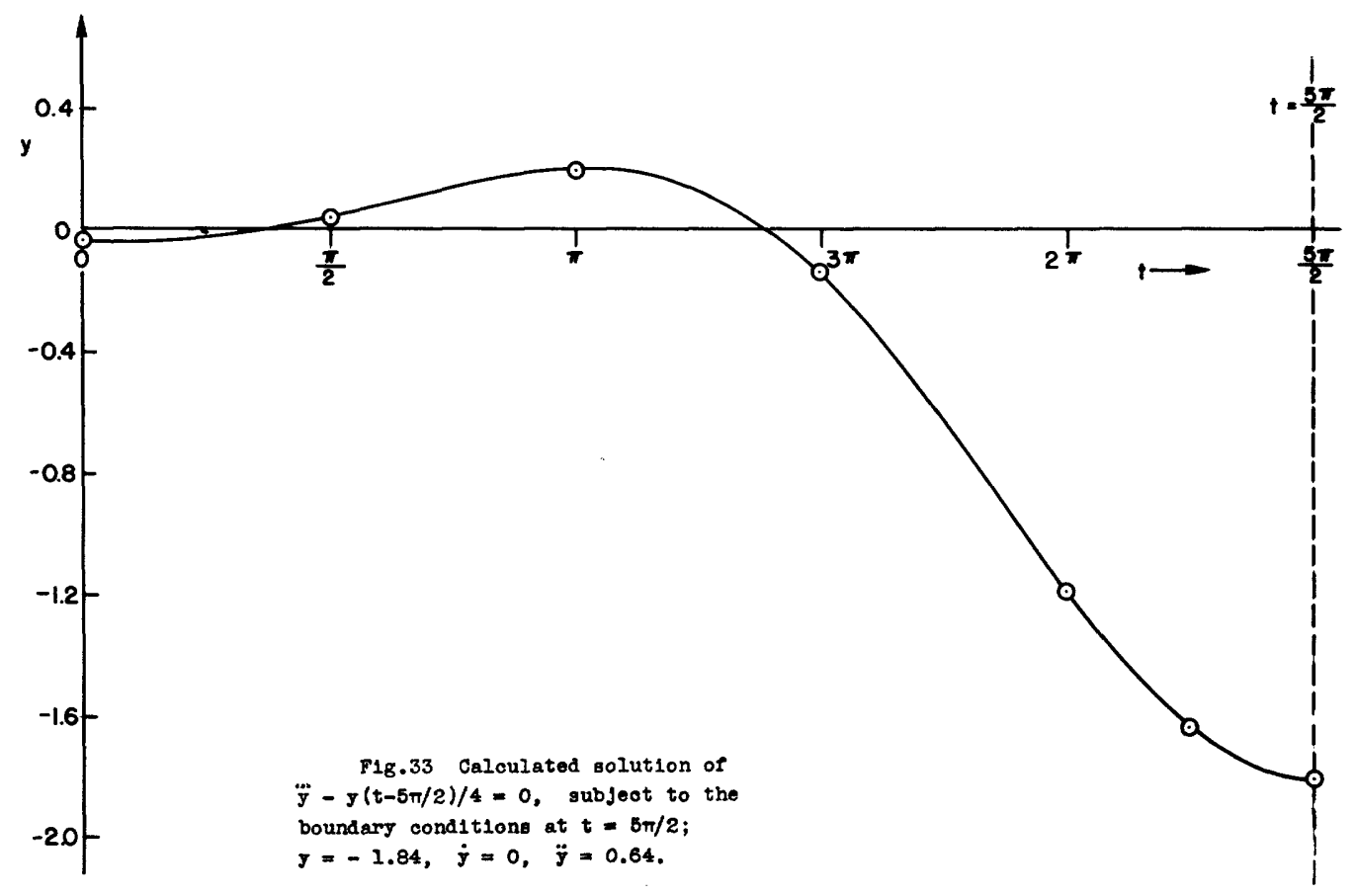

VI. SUMMARY

An electronic differential-analyzer system has been developed which is capable of handling ordinary differential equations, linear or nonlinear, and with coefficients that are either constant or variable. The components necessary to realize this system have been developed, and a small differential analyzer has been bullt, with six integrators, an arbitrary-function generator, and a high-speed multiplier. This analyzer has been used to solve a number of representative differential equations. The accuracy of this differential analyzer is limited by the cathode-ray-tube solution displays to from 1 to 5 per cent. The precision of operation, which is much more important for rapld qualitative exploration of new problems, depends upon the problem being considered but is usually of the order of one part in ten thousand.

An analysis of the influence of high- and low-frequency limitations of the differential-analyzer components has been made. This analysis permits quantitative determination of errors in the solution of ordinary differential equations with constant coefficients.

The flexibility and speed of the electronic differential analyzer permits rapid investigation of wide ranges of equation parameters and initial conditions; furthermore this speed renders practical the solution of equations for which the final rather than the initial values of the solution are known. 


\section{ACKNOWLEDGMENT}

In addition to thanks already expressed to Prof. Wallman, the author wishes to acknowledge numerous conversations with Mr. Ronald E. Scott and other colleagues in the Research Laboratory of Electronics.

The author appreciates the interest of the. Directors of the Research Laboratory of Electronics, Professors J.A. Stratton, A.G. Hill, and J.B.Weisner, under whose auspices this work has been carried out.

\section{REFERENCES}

(1) V. Bush and S. H. Caldwell, Jour. Frank. Inst. 240, 255 (1945).

(2) F. Rockett, Electronics, 21, 138 (1948).

(3) A. W. Burks, Proc. I.R.E. 35, 756 (1947).

(4) V. Bush, Jour. Frank. Inst. 212, 447 (1931).

(5) H. E. Kallman, Radiation Laboratory Internal Report 41 (1945).

(6) S. A. Wingate, "An Electronic Computing Device" Master's Thesis in Electrical Englneering, M.I.T. (1946).

(7) J. M. Ham, "A General Integrator for Electronic Analogue Computat1on", * Master's Thesis in Electrical Engineering, M.I.T. (1947).

(8) W. W. Selfert, "An Electronic Multiplier for Use in an Analyzer Computation", Master's Thesis in Electrical Engineering, M.I.T. (1948).

(9) D. M. MacKay, Nature 159, 406 (1947).

(10) D. J. Mynall, Electronic Engineer 19, 178 (1947).

(11) D. E. Sunstein, "The Photoformer", presented at the National Convention of the Institute of Radio Engineers, March, 1948.

(12) J. R. Ragazzinl, R. H. Randall and F. A. Russell, Proc. I.R.E. 35, 444 (1947).

(13) G. A. Korn, Electronics 21, 122 (1948).

(14) S. Frost, Electronics 21, 166 (1948).

(15) "Electronic Instruments", Radiation Laboratory Series, No. 21, New York McGraw-H1II (1948).

(16) J. H. Roe, Proc. I.R.E. 35, 1532 (1947). 
(17) K. R. Wendt, RCA Review 9, 85 (1948).

(18) M.I.T. Radar School Staff, "Principles of Radar", 2nd Edition, New York, McGraw-H1II (1946).

(19) N. W. McLachlan, "Theory and Application of Mathieu Functions", Clarendon Press (1947).

(20) B. Van der Pol, Proc. I.R.E. 18, 1194 (1930).

(21) W. L. Barrow, Proc. I.R.E. 21, 1182 (1933).

(22) E. Camb1, Proc. I.R.E. 36, 42 (1948).

(23) E. Jahnke and F. Emde, "Tables of Functions with Formulae and Curves", New York, Dover Publications (1943).

(24) N. Minorsky, "Introduction to Nonlinear Mechanics", Ann Arbor, J. W. Edwards (1947). 


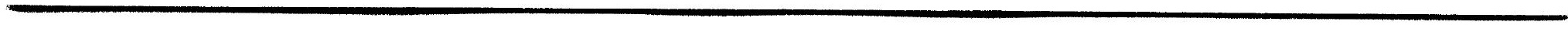

